Décadrages Décadrages

cinéma, à travers champs Cinéma, à travers champs

$18 \mid 2011$

Mario Ruspoli et le « cinéma direct »

\title{
Regard sur la folie: poétique et politique de la folie et du cinéma
}

\section{Mireille Berton}

\section{(2) OpenEdition}

1 Journals

Édition électronique

URL : https://journals.openedition.org/decadrages/216

DOI : $10.4000 /$ decadrages. 216

ISSN : 2297-5977

Éditeur

Association Décadrages

Édition imprimée

Date de publication : 10 avril 2011

Pagination : 47-68

ISBN : 978-2-9700668-2-8

ISSN : 2235-7823

\section{Référence électronique}

Mireille Berton, «Regard sur la folie : poétique et politique de la folie et du cinéma », Décadrages [En

ligne], 18 | 2011, mis en ligne le 10 avril 2012, consulté le 03 avril 2022. URL : http://

journals.openedition.org/decadrages/216; DOI : https://doi.org/10.4000/decadrages.216 


\title{
Regard sur la folie: poétique et politique
}

\author{
de la folie et du cinéma
}

par Mireille Berton

Souvent perçu comme un discours édifiant sur l'horreur de la folie, le film de Ruspoli doit être avant tout appréhendé dans le contexte de la psychiatrie institutionnelle (mieux connue, dès mai 1968, sous le nom d'anti-psychiatrie) qui lutte, depuis la fin de la Seconde Guerre mondiale, pour le désenclavement de la logique asilaire classique. Ce mouvement psychiatrique s'engage alors, sur la base de courants de pensée issus du surréalisme, du marxisme et de la psychanalyse, contre l'exclusion et l'avilissement des fous. En consonance parfaite avec cette psychiatrie politisée, Regard sur la folie (Mario Ruspoli, France, 1962) met en œuvre sur le plan cinématographique certaines des valeurs prônées par une approche humaniste du fou, dont celle de la fluidité et de la créativité de la parole comme lieu d'une "vérité» à écouter. Situé au cœur d'un réseau intellectuel, humain et artistique extrêmement dense, ce film, tourné à Saint-Alban avec la collaboration des psychiatres de l'hôpital, doit être considéré comme la trace historique d'une conjonction entre deux champs - le cinéma direct et la psychiatrie institutionnelle - qui partagent une sensibilité commune de la folie inaugurée dans l'orbite surréaliste. Cet article se propose de restituer l'histoire de cette conjonction, avec comme fil rouge l'hypothèse d'une dimension «surréalisante» du film sur laquelle l'historiographie du cinéma direct semble avoir fait l'impasse.

«Et quelle incarcération! On sait - on ne sait pas assez - que les asiles, loin d'être des asiles, sont d'effroyables geôles, où les détenus fournissent une main-d'œuvre gratuite et commode, où les sévices sont la règle, et cela est toléré par vous. L'asile d’aliénés, sous le couvert de la science et de la justice, est comparable à la caserne, à la prison, au bagne. [...] Nous d'admettons pas qu'on entrave le libre développement d'un délire aussi légitime, aussi logique que toute autre succession d'idées ou d'actes humains. La répression des réactions antisociales est aussi chimérique qu'inacceptable en son principe. Tous les actes individuels sont antisociaux. Les fous sont les victimes individuelles 
1 "Lettre aux médecins-chefs des asiles de fous" [texte collectif], in La Révolution surréaliste, $\mathrm{n}^{\circ} 3$, 1925, repris dans Maurice Nadeau, Histoire du surréalisme, suivi de Documents surréalistes, Paris, Seuil, 1964, p. 213.

2 Entre 1941 et 1945, plus de 40000 malades mentaux sont en effet décédés dans un état de misère et de famine catastrophique. SaintAlban, non seulement sera l'une des rares exceptions (aucun de ses malades ne mourra durant cette période), mais servira de refuge aux clandestins, intellectuels et artistes, qui côtoieront les malades internés pour échanger soins et idées. Voir: Max Lafont, L'extermination douce. La mort de 40000 malades mentaux dans les hôpitaux psychiatriques en France, sous le régime de Vichy, préf. de Lucien Bonnafé et Claude David, Nantes, Ed. de I'AREFPPI, 1987. Pour prendre la mesure de la crise que traverse la psychiatrie française après le Seconde Guerre mondiale, je renvoie au numéro de la revue Esprit: "Misère de la psychiatrie", décembre 1952 (articles de Ph. Koechlin, L. Bonnafé, H. Ey, Fr. Tosquelles, etc.). Ce numéro contient des articles qui font le bilan de l'état des hôpitaux psychiatriques d'après-guerre, qui proposent des mesures pour transformer la psychiatrie et qui livrent le point de vue des malades eux-mêmes, notamment grâce à des textes poétiques, l'écriture ou l'expression artistique étant l'une des composantes de la thérapeutique préconisée par ces psychiatres. Pour une excellente introduction à I'histoire de la psychiatre en France, voir le documentaire Histoires autour de la folie, de Paule Muxel et Bertrand de Solliers, Editions Montparnasse, 2006.

3 Terme emprunté à Erving Goffman: Asiles: études sur la condition sociale des malades mentaux et autres reclus, trad. de Liliane et Claude Lainé; présentation de Robert Castel, Paris, Les Editions de Minuit, 1968: "On peut définir une institution totalitaire (total institution) comme un lieu de résidence et de travail où un grand nombre d'individus, placés dans la même situation, coupés du monde extérieur pour une période relativement longue, mènent ensemble une vie recluse dont les modalités sont explicitement et minutieusement réglées " (p. 41). par excellence de la dictature sociale; au nom de cette individualité qui est le propre de l'homme, nous réclamons qu'on libère ces forçats de la sensibilité, puisqu'aussi bien il n'est pas au pouvoir des lois d'enfermer tous les hommes qui pensent et agissent.»

Signée en 1925 par les membres du mouvement surréaliste, cette "Lettre aux médecins-chefs des asiles de fous" dénonce, simultanément, l'état désastreux des institutions asilaires qui refoulent leurs malades à la périphérie de la société et la dépréciation du fou considéré comme un individu de seconde zone $\mathbf{1}$. Valorisant la folie comme une forme de créativité qui rend justice à la liberté humaine et à ses potentialités multiples, les surréalistes envisagent le trouble psychique dans sa valence révolutionnaire, en tant qu'il peut remettre en question l'ordre établi en imposant une vision du monde en prise directe avec une réalité qui échappe à la plupart des êtres socialement adaptés. Le fou sait plus, voit mieux, exprime au-delà des conventions sociales, des normes morales et de la logique avérée par l'expérience quotidienne. Le fou mérite par conséquent qu'on le respecte, qu'on le soutienne et qu'on l'écoute dans son délire même, ce lieu propice à la fantaisie, à l'imaginaire et à la subversion (fig. 1).

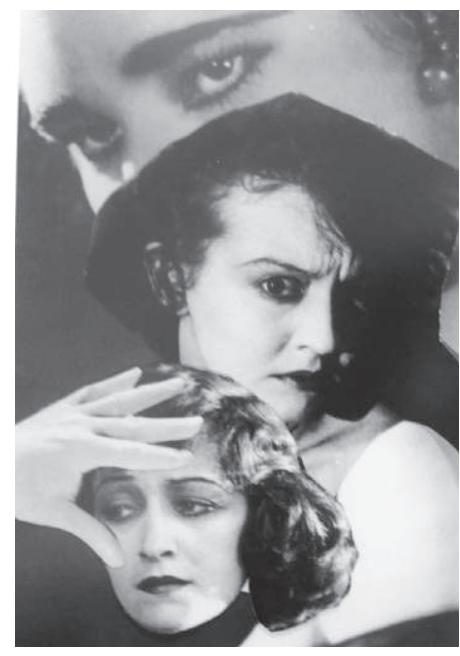

1/ Paul Eluard, L'Hystérie, 1938

\section{La psychiatrie institutionnelle: une politique de la folie}

Une vingtaine d'années plus tard, juste après la Seconde Guerre mondiale, l'opinion publique découvre qu'en France environ 40000 pensionnaires d'hôpitaux psychiatriques sont morts de faim, de misère et de froid dans des conditions effroyables ${ }^{2}$. Comparée par certains contemporains aux camps de concentration nazis, l'«institution totalitaire $\mathbf{3}$ que représente 
l'asile psychiatrique classique va connaître dans l'après-guerre une crise sans précédent, entraînant du même coup une vague de réflexions et de réformes dont la psychiatrie institutionnelle est la plus représentative $\mathbf{4}$. Des psychiatres et des infirmiers se mobilisent en différents lieux et de manière non concertée pour repenser leur pratique au sein d'institutions dont il s'agit de proscrire le caractère concentrationnaire (ordre, enfermement, ségrégation, servilité, médicalisation à outrance, etc.), afin de redonner au patient une valeur et une dignité humaines. La clinique de la Borde avec Jean Oury et Félix Guattari, l'hôpital de Fleury-lesAubrais avec Georges Daumézon et Philippe Koechlin et, surtout, l'hôpital de Saint-Alban en Lozère autour de Lucien Bonnafé et François Tosquelles, constituent les centres névralgiques d'un mouvement conjuguant pratique thérapeutique, recherche théorique, formation du personnel et action politique - mouvement qui sera baptisé en mai 1968 d'anti-psychiatrie et qui aura un impact sans précédent sur la psychiatrie de la seconde moitié du XX ${ }^{\mathrm{e}}$ siècle (fig. 2) ${ }^{5}$.

Brisant le carcan imposé par une psychiatrie sclérosée et déshumanisante, ces médecins, nourris par la pensée de Freud, Lacan et Marx, issus de la Résistance et inspirés par l'idéologie révolutionnaire et poétique

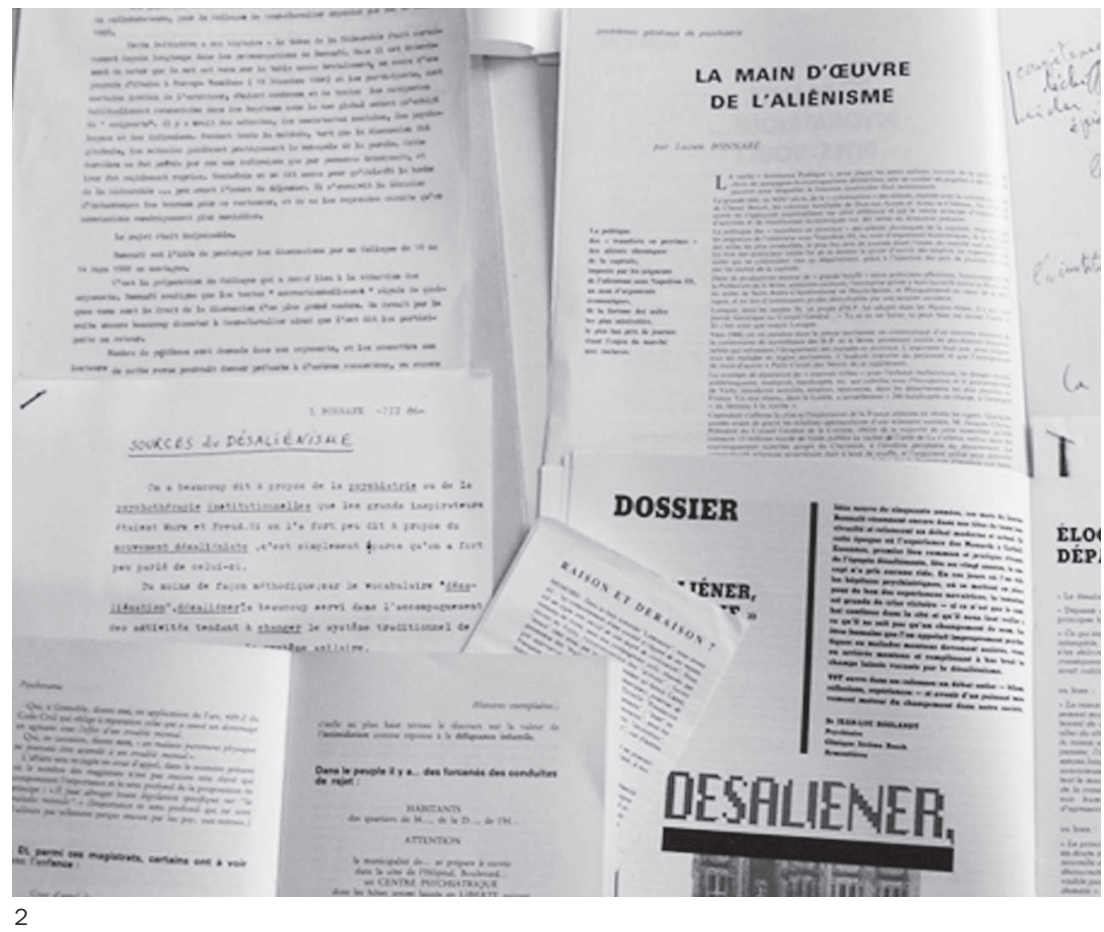

4 Cette dénomination de "psychiatrie institutionnelle " apparaît pour la première fois dans un article signé par Philippe Koechlin et Georges Daumezon, "La psychothérapie institutionnelle française " publié dans Anais Portugueses de psiquiatria (vol. 4, n 4 , décembre 1952, pp. 272311). Elle désigne (en partie rétrospectivement) une pratique psychiatrique novatrice qui s'est développée depuis le Seconde Guerre mondiale, notamment autour de I'hôpital de Saint-Alban en Lozère où sera tourné Regard sur la folie. Ce mouvement cherche à remettre en question l'organisation asilaire en ôtant les barreaux aux fenêtres, en ouvrant les espaces, en impliquant les malades dans des assemblées décisionnelles et en libérant les infirmiers de leur rôle strict de gardiens de "prisons" pour aliénés.

5 N'en déplaise à Jacques Hochmann qui expédie en quelques pages, dans son Histoire de la psychiatrie, le chapitre sur la psychiatrie institutionnelle, dont les bases théoriques sont jugées "vagues" hormis l'évidence d'un "fond marxisant." (Jacques Hochmann, Histoire de la psychiatrie, Paris, PUF, coll. Que sais-je $\mathrm{n}^{\circ} 1428$, 2006 [2004], p. 92). Pour des synthèses plus conséquentes émanant des protagonistes du mouvement, se référer à: Jean Oury et Ginette Michaud, "Psychothérapie institutionnelle. Une introduction" [en ligne], www.euro-psy.org/site/ La.../psy_insti_oury-michaud.pdf (consulté le 29 juin 2010); Jean Oury, "Psychanalyse, psychiatrie et psychothérapie institutionnelles", in Vie sociale et traitements, 2007/3, $\mathrm{n}^{\circ}$ 95, pp. 110-125. Et aussi: Jean-Michel Jamet, "De l'asile à la psychothérapie institutionnelle. $\mathrm{Ou}$ comment l'économie saborde le soin " [en ligne], www.serpsy.org/histoire/jamet.html, consulté le 30 juin 2010). Voici la définition que donnent Oury et Michaud de la psychiatrie institutionnelle: "I s'agit d'abord de soigner) collectivement ces lieux de ségrégation par une nouvelle structure des rapports humains, en attribuant à chaque malade une responsabilité dans des tâches très variées et en favorisant le remaniement et l'ouverture des systèmes de partage du collectif: création de groupes d'ergothérapie, groupes de jeux ou de théâtre, ouverture du quartier et de l'hôpital, etc. C'est ainsi que se constitue à l'intérieur de l'établissement, un réseau très précis d'institutions dont les caractéristiques sont la souplesse, la mouvance, voire même la précarité, afin de s'adapter aux demandes qui se font jour, que ce soit à partir des individus, des relations inter-individuelles ou de groupes. II s'agit donc de créer un ensemble structural permettant à chacun de pouvoir s'investir vis-à-vis d'‘objets institutionnels', seuls supports possibles du transfert chez les malades psychotiques". 
6 Les différentes structures et personnalités qui composent l'institution psychiatrique - et la variabilité des fonctions des unes et des autres en son sein - favorisent chez le patient la libre circulation de désirs et de fantasmes qui se jouent une "scène" partagée et organisée collectivement, ce qui présente l'avantage de reconnaître au malade la possibilité d'une symbolisation, d'une reconnaissance et d'une interprétation de sa souffrance. La psychothérapie institutionnelle s'appuie sur la notion de transfert telle qu'élaborée dans le champ psychanalytique. D'après le Vocabulaire de la psychanalyse de Laplanche/Pontalis, le transfert désigne "le processus par lequel les désirs inconscients s'actualisent sur certains objets dans le cadre d'un certain type de relation établi avec eux et éminemment dans le cadre de la relation analytique. II s'agit là d'une répétition de prototypes infantiles vécue avec un sentiment d'actualité marqué. [...] Le transfert est classiquement reconnu comme le terrain où se joue la problématique d'une cure psychanalytique, son installation, ses modalités, son interprétation et sa résolution caractérisant celle-ci " (Vocabulaire de la psychanalyse, Paris, Quadrige/PUF, 1998 [1967], p. 492).

7 Voir par exemple: Claude Beylie, "La nature crie (Regard sur la folie)", in Cahiers du cinéma, novembre 1962, $n^{\circ} 137$, pp. 53-56. L'auteur met en évidence le caractère aride d'une démarche filmique avide de vérité brute: "La vérité, aujourd'hui, il est clair qu'il faut l'aller chercher dans le lieu le plus abrupt, l'être le plus déshérité, chez les gens de la terre ou bien ceux, si proches de l'asile, qui conçoivent Paris comme une fournaise au loin [...]" (p. 54). Déplorant "ce profil de fou découpé dans la pénombre de l'asile, cherchant dérisoirement à exprimer quelque chose par le langage des mots", Beylie emploie les termes dépréciatifs de "viol", de "maculature", de "goût amer de la vérité " pour qualifier le travail d'une équipe qui "a filmé des kilomètres de pellicule dont il fut extrait par la suite non pas forcément le meilleur, ce qui aurait été déjà une notable trahison, mais du moins, le plus (direct), le plus bredouillé, le plus humain en somme, si ce mot offre encore quelque sens - disons le plus digne de nous faire aimer ces hommes-là" (id.). Plus loin, il définit le film comme une "expérience inouïe de ce qui relève assurément de quelque inframonde, pathétique plongeon dans la nuit très obscure où l'âme subit une totale érosion, démarche hagarde et pour ainsi dire
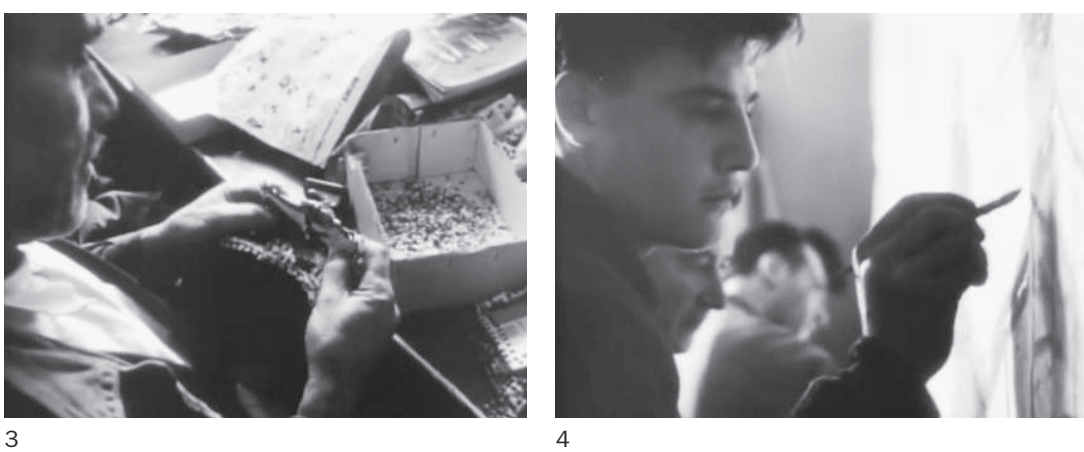

du surréalisme, proposent une nouvelle organisation des rapports entre soignants et soignés, entre hôpital et société, et ce dans le but d'abolir les hiérarchies internes, de conférer un rôle actif au malade et au personnel et de décloisonner les espaces physiques et mentaux. Cherchant à renouveler les rapports sociaux, la psychiatrie institutionnelle mise notamment sur les effets bénéfiques de dynamiques de groupes via la participation de chacun des résidents à des commissions, bureaux, activités culturelles, ateliers ergothérapeutiques ou clubs de discussion qui fonctionnent comme autant de lieux de symbolisation (fig. 3 et 4). Partie prenante du processus curatif qui exige et postule la désaliénation de l'institution elle-même, l'hôpital s'ouvre alors sur l'extérieur, à savoir sur les habitants du village environnant, les administrations, les services sociaux, la gendarmerie, les églises, etc., tissant autour du malade un réseau relationnel et intersubjectif prêt à offrir au psychotique une vaste palette de mécanismes transférentiels nécessaires à sa guérison ou, du moins, à son bien-être ${ }^{6}$. A l'opposé du patient névrotique qui parvient à symboliser son symptôme via des processus de refoulement et d'identification à l'image de l'autre (jouant ainsi son mal sur les registres de l'Imaginaire et du Symbolique), le psychotique - victime d'un transfert dissocié et d'une très grande difficulté à symboliser sa souffrance (ce qui l'amène à interagir directement avec le registre du Réel) - doit être traité de manière à lui proposer un maximum de surfaces projectives qui vont lui redonner un sentiment d'appartenance au monde. D'où la nécessité de faire circuler autant que possible la parole (donc le désir, le fantasme) d'un individu à l'autre, d'affecter chacun des participants tour à tour à des "rôles» en constante mutation, de manière à garantir les préceptes d'une psychiatrie psychanalytique qui se veut avant tout modulable, égalitaire et communautaire.

En 1961, Regard sur la folie de Mario Ruspoli s'emploie précisément à donner de cette forme de psychothérapie collective une représenta- 
tion qui prenne en considération son originalité et sa spécificité, notamment en articulant le texte filmique à la parole du fou. Mal compris à sa sortie par certains critiques de cinéma qui l'interprètent comme un portrait glacial et sinistre du malade mental et de l'institution qui l'abrite 7 , le film puise pourtant toute sa force dans sa capacité à renvoyer directement ou indirectement au carrefour extraordinaire d'idées et de personnes qui se sont croisées à Saint-Alban entre 1940 et 1960 autour de valeurs communes: la solidarité avec les malades délaissés et méprisés par les pouvoirs publics, la résistance contre les totalitarismes, la contestation du système capitaliste, la collectivité comme alternative à l'organisation verticale des pouvoirs, la créativité comme modalité existentielle et thérapeutique. Différentes personnalités - des médecins, des infirmiers, des philosophes, des résistants, des artistes, etc. - contribuent en effet à créer un faisceau d'influences où se mêlent psychanalyse, militantisme et surréalisme, le cinéma participant sous différentes formes, comme on le verra plus loin, à une réflexion de fond sur le sens d'une pratique médicale ardue et souvent méconnue. C’est ce réseau intellectuel, artistique et humain dont le film de Mario Ruspoli se fait l'écho que je me propose de reconstituer dans les lignes qui suivent.

\section{Un film surréaliste?}

Débutant, dans la foulée du pré-générique et du générique, sur un travelling parcourant les couloirs de l'hôpital de Saint-Alban - mouvement de caméra accompagné d'un texte d'Antonin Artaud récité en voix off par Michel Bouquet ${ }^{8}$-, Regard sur la folie se place d'emblée sous l'égide du

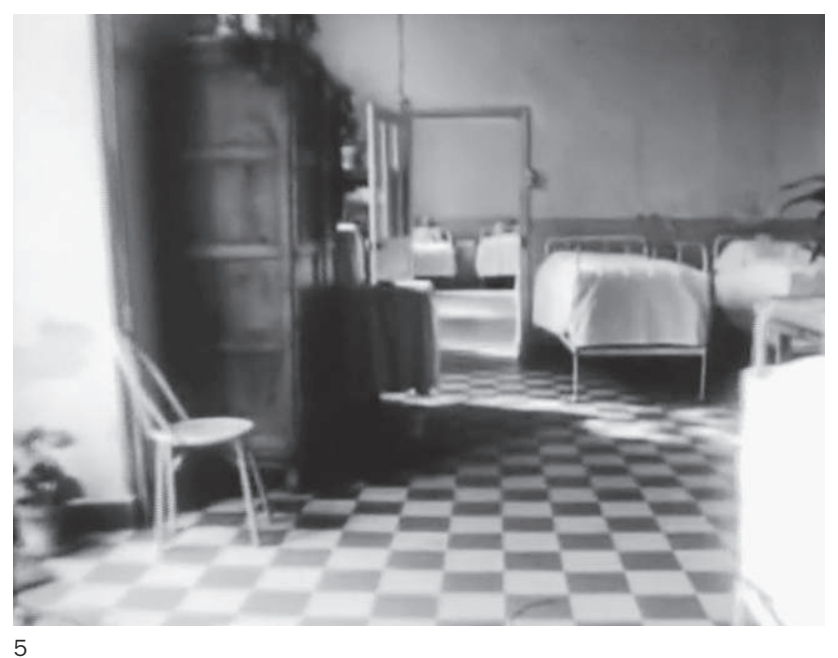

sisyphéenne de ces fantoches errant dans le brouillard froid du jardin de l'asile [...]" (p. 56). Sur un ton solennel, un rédacteur du Canard enchaîné écrit: "Témoignages atroces, pathétiques, insoutenables. Face à ces malheureux, des infirmiers pas toujours compréhensifs et des médecins qui essaient de comprendre, ce qui ne facilite pas le dialogue." (19 septembre 1962, signé J.-P.G). A l'opposé, Raymond Bellour indique la "qualité de regard peu commune, à la fois curieux, ému, exalté, attendri [...]" de Ruspoli qui "a su échapper par moment à l'emprise de la réalité à laquelle il a choisi d'être tenu, il a pris sa distance, s'est fait juge, regard, miroir. Tout simplement en laissant errer l'image et en la clouant à sa volonté par le texte [...]. " (Raymond Bellour, "Un cinéaste-vérité", in Esprit, avril 1962, n 4, pp. 625-626). Si les critiques de la grande presse mettent en exergue le caractère cru et impitoyable des images, ils se montrent globalement admiratifs (Le Monde, L'Express, L'Humanité, France observateur, Le Canard enchaîné, Le Figaro, etc.), louant la nature bouleversante et poignante, à la fois humaniste, tendre et sans complaisance de ces portraits de fous: le film proposerait ainsi "des images d'aujourd'hui, nettes, crues, désagréables parfois, obsédantes toujours, inoubliables [...]. Les images sont parfois insoutenables, mais il se dégage de tout cela une telle tendresse, une telle lucidité qu'on ne peut s'empêcher d'admirer le talent, le courage du cinéaste et de son équipe pour les documents qu'ils offrent à notre méditation." (Samuel Lachize dans L'Humanité, 17 décembre 1962); Robert Benayoum dans France Observateur note: "Nous assistons à des confrontations bouleversantes entre psychiatres et malades, à une sorte de mise à nu qui nous concerne presque trop, tant elle souligne l'aliénation réelle, à un degré tout autre de l'individu <normal . [...] L'expérience de Ruspoli passionne, déconcerte et interroge. " (20 septembre 1962); Seule la Tribune de Genève signale que SaintAlban met en œuvre des "méthodes originales de rééducation" " Regard sur la folie: Film privé seulement?", in Tribune de Genève, 17 janvier 1963, signé C. M.) (Dossier de presse n 1859 , Cinémathèque suisse).

8 En 1955, Alain Resnais, dans Nuit et Brouillard, recourt au même procédé (travelling avec un texte de Jean Cayrol dit par Michel Bouquet), confirmant cette corrélation entre camp de concentration et asile psychiatrique qui s'établit si souvent dans l'esprit des contemporains d'après-guerre. 
9 Cette méprise sur l'origine du texte utilisé dans cette séquence - et qui a été choisi par les psychiatres de Saint-Alban (François Tosquelles, Yves Racine, Roger Gentis) qui ont collaboré au film conformément à leur "ascendance" surréaliste ici postulée - proviendrait du cinéaste lui-même qui se serait exprimé làdessus dans un entretien. Seul Claude Mauriac, dans sa critique cinématographique du Figaro littéraire (29 septembre 1962), fait référence à la bonne source. Une connaissance un peu rigoureuse de l'œuvre d'Antonin Artaud ne permet pourtant pas de confondre ses lettres des années 1940 avec celles des années 1920, les Lettres de Rodez, rédigées à la fin de sa vie lors de son internement sous la supervision du $D^{r}$ Gaston Ferdière, témoignant d'un délire sous-tendu par un mysticisme et un puritanisme absents vingt ans plus tôt. Ce n'est pas par hasard si les médecins choisissent précisément des extraits de lettres contemporaines de textes en prose de la période dite surréaliste (L'Ombilic des limbes, Le Pèse-nerfs, etc.) puisqu'ils partagent avec ceux-ci une affinité stylistique et sémantique évidente. Pour s'en convaincre, il suffit de se rapporter à l'édition de L'Ombilic des limbes chez Gallimard (coll. Poésie) qui fait précéder ces textes de la Correspondance à Jacques Rivière (L'Ombilic des Limbes, suivi de Le Pèse-nerfs et autres textes, Paris, Gallimard, 1989 [1968]). Cette erreur continue d'être répétée de nos jours dans le cadre de présentations accompagnant la projection du film dans des festivals ou dans des ouvrages sur le cinéma direct. Gilles Marsolais, par exemple, écrit: "Mario Ruspoli n'a cerné que des délires marginaux, il n'a pas montré la totalité de la réalité asilaire qui est parfois, par plusieurs aspects, sordide. En ne cernant que le phénomène de la folie douce, il rend cette maladie émouvante, voire poétique. Le texte d'Antonin Artaud, sur l'angoisse, extrait des Lettres de Rodez, si <vrai > et descriptif soit-il, ne peut que raffermir ce sentiment chez le spectateur non averti. " (Gilles Marsolais, L'aventure du direct, Paris, Cinéma club/Seghers, 1974, p. 236).

$10 \mathrm{~A}$ propos de la maladie d'Artaud, de ses internements et de son "écriture symptomatique" (S. Harel), voir: Simon Harel, "Créativité et symptomatologie dans la correspondance d'Artaud avec Jacques Rivière", in Etudes littéraires, vol. 22, n 2, 1989, pp. 73-86; André Roumieux, Artaud et l'asile, Paris, Séguier, 1996. surréalisme, mouvement littéralement hanté par la poétique de la folie (fig. 5). Repérés à tort par l'historiographie classique du cinéma direct dans les Lettres de Rodez 9 , ces extraits sont tirés de fait de deux sources : la correspondance d'Artaud avec Jacques Rivière, à savoir ses lettres du 29 janvier 1924 et du 25 mai 1924, et L'Ombilic des limbes (plus précisément son texte "Description d'un état physique», 1925). Relatifs à une époque où il est encore un membre actif et nodal du groupe surréaliste, les propos d'Artaud (qui souffre depuis son adolescence de troubles psychotiques allant en s'aggravant 10) apparaissent tels quels dans la bande-son du film :

"Un effondrement central de l'âme, [...] une espèce d'érosion, essentielle à la fois et fugace, de la pensée, [...] la séparation anormale

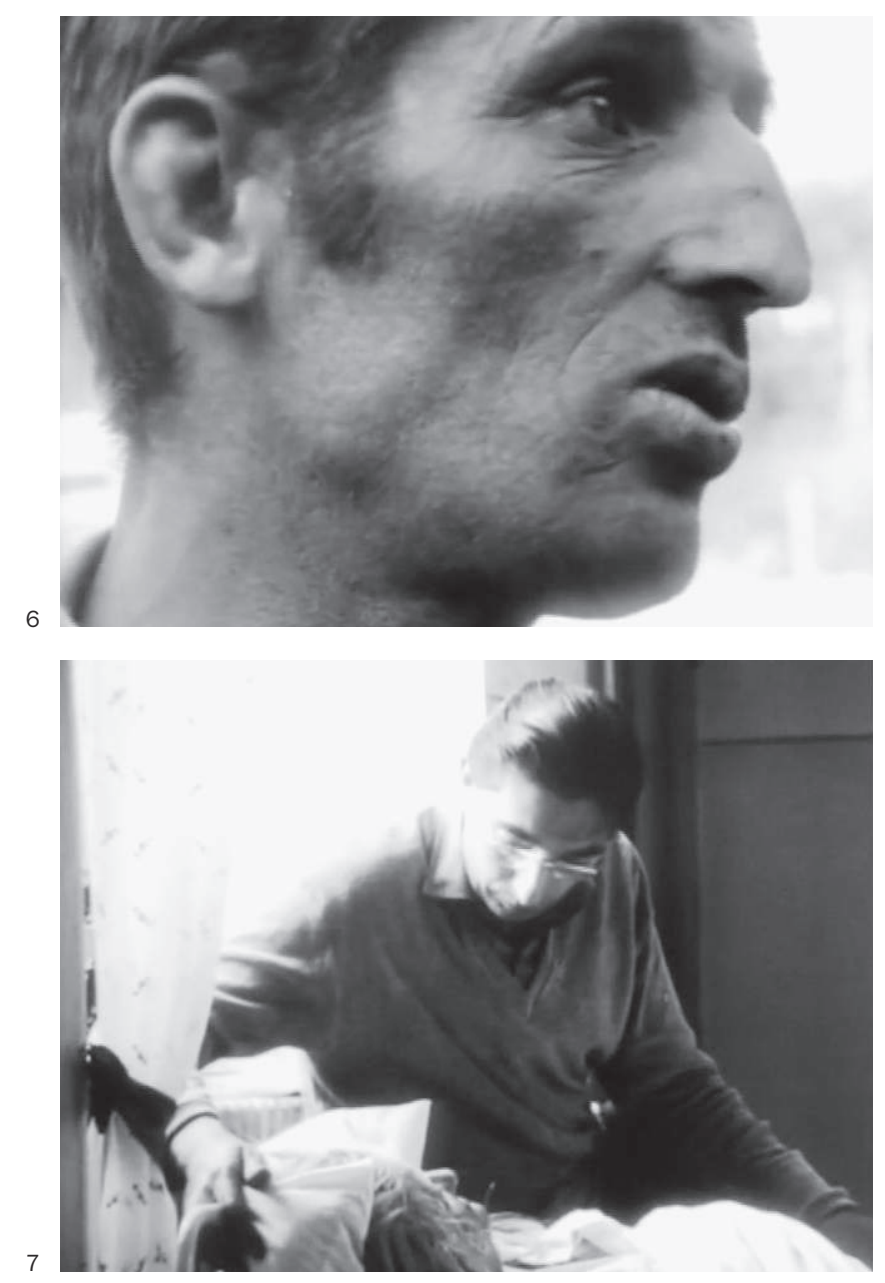


des éléments de la pensée [...]11. / Une maladie qui affecte l'âme dans sa réalité la plus profonde, et qui en infecte les manifestations. Le poison de l'être. Une véritable paralysie. Une maladie qui vous enlève la parole, le souvenir, qui vous déracine la pensée 12. / Une fatigue renversante et centrale, une espèce de fatigue aspirante. [...] Une fatigue de commencement du monde, la sensation de son corps à porter, un sentiment de fragilité incroyable, et qui devient une brisante douleur. $\mathbf{1 3}$

Ces passages font évidemment écho à toutes les prises de paroles des patients interrogés par l'équipe du tournage ou écoutés par les médecins, à commencer par celle de Riquet (l'homme filmé en gros plan dans le prologue) qui répond aux questions de Dolorès Grassian : la folie attaque l'être dans ses fondamentaux: la pensée, le langage, la mémoire; elle lui fait perdre le sens d'identité humaine, elle l'arrache à ses semblables, elle lui impose le poids d'une perte et d'une solitude sans remèdes (fig. 6). Une autre malade, $\mathrm{M}^{\mathrm{me}}$ Blanche, assistée par un psychiatre assis au bord du lit, reformule cette vision de la folie comme une chute et un basculement dans le vide aspirant. Le dialogue entre médecin et patiente tente alors de renouer les fils d'une histoire personnelle à l'origine d'une douleur actuelle, la bienveillance, l'empathie et la familiarité du soignant sans uniforme tentant de sécuriser une malade fragilisée par des peurs et une confusion d'idées contradictoires (fig. 7). L'image se désolidarise un temps du discours pour montrer en contre-point comment le psychotique tisse dans le vide, à coup de gestes répétitifs, un monde qu'il tente en vain d'habiter (fig. 8). Ainsi, la folie telle qu'elle apparaît dans
11 Antonin Artaud, "Lettre d'Antonin Artaud à Jacques Rivière, Paris, le 29 janvier 1924", in CEuvres complètes, tome 1, Lettres à Jacques Rivière, Paris, Gallimard, 1971, p. 25.

12 Antonin Artaud, "Lettre d'Antonin Artaud à Jacques Rivière, Paris, le 25 mai 1924 ", in CEuvres complètes, id., p. 40.

13 Antonin Artaud, "Description d'un état physique", in L'Ombilic des limbes, suivi de Le Pèse-nerfs et autres textes, op. cit., p. 62.

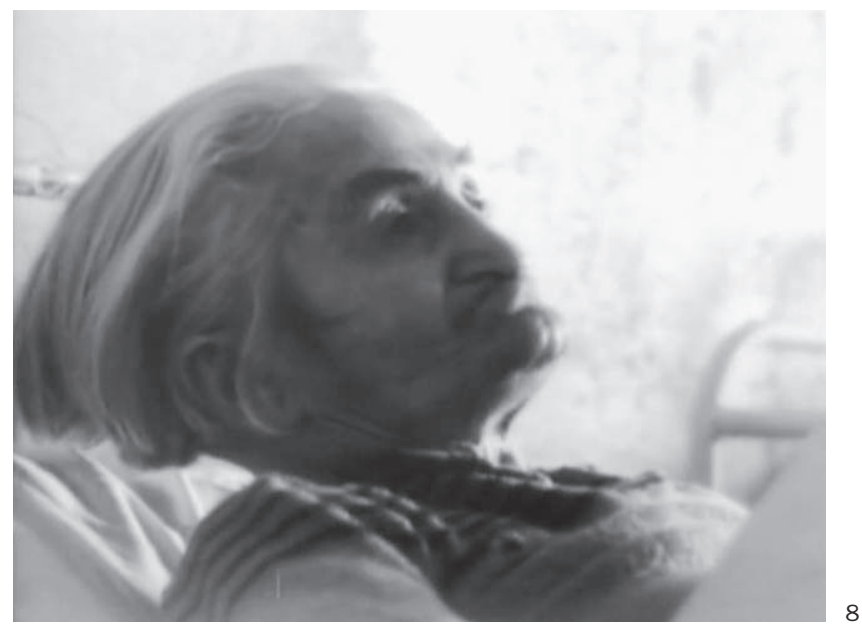


14 "[...] Le film ne donne pas une vision complète de l'hôpital: les traitements physiques et chimiques modernes, largement utilisés ici comme partout, les soins de toute nature dispensés à l'extérieur dans un ensemble d'institutions en plein développement, n'y sont pas abordés. Nous n'avons pas voulu rassurer le public par des images souriantes, et il en est, d'un établissement moderne sans murs ni grilles [...]".

15 Extrait du commentaire en voix off. ce film n'est ni douce ni dure, comme le suggère Marsolais, mais se présente avant tout dans sa vérité profonde : le fou c'est celui qui a perdu le contact avec la réalité immédiate. Pour pallier cette perte, il en imagine alors une autre qui l'enferme dans un délire, tout en le protégeant du monde extérieur devenu, à son tour, insensé.

Si le film, en effet, comme l'indique le carton d'introduction ${ }^{\mathbf{1 4}}$, ne livre ni une image exhaustive et didactique de la vie en institution psychiatrique et de l'arsenal de soins employés pour soulager les malades, ni un portrait idéalisé des personnes souffrant de troubles psychiques, il met en scène les difficultés du fou à se connecter à son milieu et le moyen pour parvenir, malgré cet obstacle, à créer du lien à travers le langage, la parole, l'écriture. Le «fou-poète» qui récite ses vers lors des assemblées réunissant périodiquement médecins, patients, infirmiers, sœurs et invités de passage, emblématise parfaitement une vision de la folie commune à l'équipe soignante et à l'équipe de filmage: c'est dans l'expression artistique/discursive que le malade retrouve un peu de son identité humaine, les différents ateliers organisés à Saint-Alban encourageant les patients à «briser les murs intérieurs de la folie» $\mathbf{1 5}$. Le "poète" de Saint-Alban représente, par sa créativité, son discours réfléchi, sa pensée raffinée et sa révolte intérieure, une sorte de double d'Antonin Artaud qui ne cessera, lui aussi, d'appeler à une reconnaissance de son statut d'homme et d'artiste (fig. 9). La psychiatrie institutionnelle inaugure en effet une pratique qui consiste à respecter le fou dans son malêtre en tentant de valoriser chez lui une capacité de décision, d'initiative et d'inventivité totalement brimée dans les anciennes institutions.

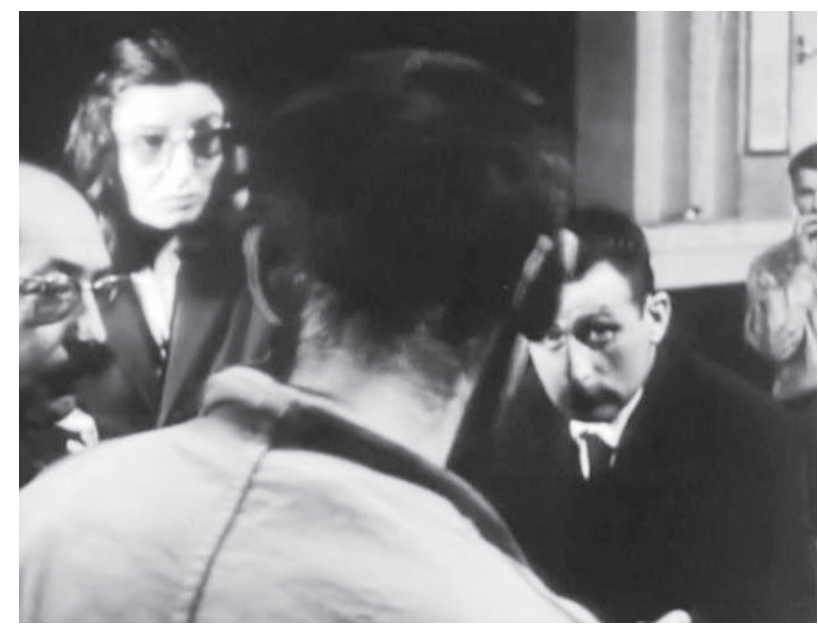

9 


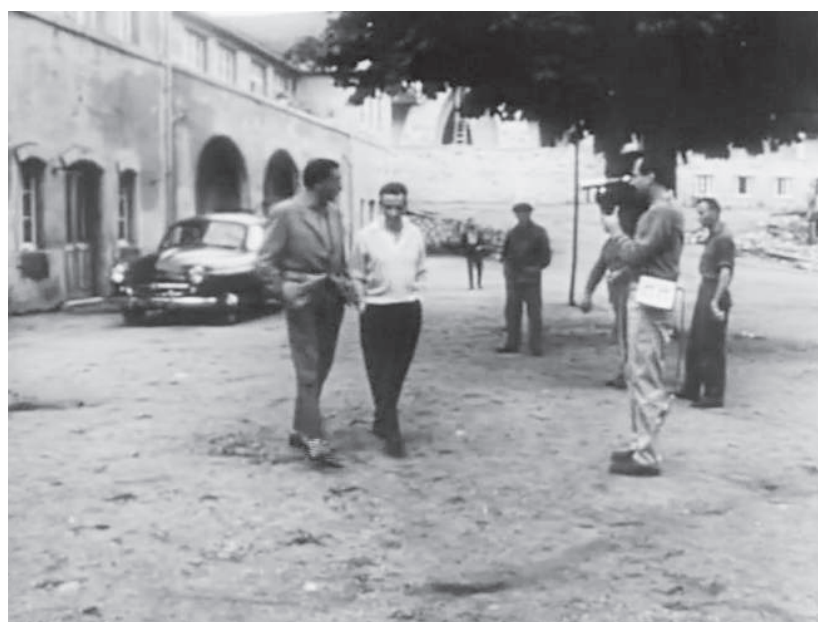

10

Le modèle de la folie surréaliste fait donc retour à travers la figure du poète qui ponctue le film de ses interventions jusqu'à La Fête prisonnière, son second volet dont le commentaire conclusif réitère la définition de la folie comme solitude - une solitude que seul le rapport avec d'autres êtres humains, fous ou non, est en mesure d'atténuer. Le plan final, qui montre Michel Brault suivant la marche de deux psychiatres conversant entre eux (fig. 10), suggère combien l'acte de filmage peut œuvrer comme une opération "suturant» les fragments de subjectivité éparses au gré des errances individuelles. Contrairement à ce qu'avancent les critiques du film qui dénoncent sa faiblesse à trop vouloir esthétiser la folie via une image édulcorée et partielle de la réalité asilaire ${ }^{\mathbf{1 6}}$, Regard sur la folie doit être saisi avant tout comme un film "surréalisant» et militant qui rend compte d'une pratique inédite, celle de la psychiatrie institutionnelle dont les liens historiques avec le surréalisme et, partant, un engagement politique et une réflexion théorique en consonance avec celui-ci, sont attestés sur différents plans.

La maigre littérature secondaire existant sur le film ${ }^{\mathbf{1 7}}$ ne cesse en effet de s'interroger sur son adéquation à une réalité donnée, les uns accusant Ruspoli de voyeurisme sordide, les autres de «rigueur quasi ascétique» $\mathbf{1 8}$ ou alors de condescendance doublée de formalisme, oubliant de situer Regard sur la folie dans son contexte social, historique et culturel, à savoir celui du mouvement de réforme asilaire qu'entreprennent les partisans d'une psychiatrie remettant en question la vision bourgeoise de la folie ${ }^{19}$, revendication, rappelons-le encore, déjà formulée par les surréalistes. Reprenons donc l'histoire de cette filiation entre
16 A l'opposé de Claude Beylie cité plus haut, Gilles Marsolais estime que Ruspoli a voulu donner une image lyrique et sentimentale de la folie, passant ainsi à côté de sa réalité triviale: "En voulant abolir certains mythes et détruire certains clichés ancrés dans l'esprit du public à propos de cette maladie, Mario Ruspoli a faussé quelque peu le portrait sociologique qu'il aurait pu nous offrir. L'inquiétude qui naît parfois de l'œuvre se transmue aussitôt en poésie ou en plaisir visuel. C'est dommage." (Gilles Marsolais, L'aventure du direct, op. cit., p. 236). Plus tard, dans son ouvrage L'aventure du cinéma direct revisitée, Marsolais modifie à peine sa lecture du film, hormis pour ajouter qu'il "est tiraillé entre le regard clinique et le point de vue personnel" et que "la beauté visuelle de ce film [...] n'est pas exempte d'une certaine complaisance." (Gilles Marsolais, L'Aventure du cinéma direct revisitée, Laval, Les 400 coups, 1997, p. 138).

17 Elle se résume à peu de choses, et notamment à des articles ou des passages dans des ouvrages: Raymond Bellour, "Un cinéastevérité", op. cit.; Claude Beylie, "La nature crie (Regard sur la folie)", op. cit.; Mouloud Boukala, "Un dispositif d'énonciation - ‘Faire comme si ’, avec Mario Ruspoli ", in Le dispositif cinématographique, un processus pour [re]penser l'anthropologie, Paris, Téraèdre, 2009, pp. 107122; Guy Gauthier, Le documentaire, un autre cinéma, Paris, Nathan 1995, passim; Guy Gauthier, Philippe Pilard, Simone Suchet, "Mario Ruspoli ", in Le Documentaire passe au direct, Montréal, VLB Editeur, 2003, pp. 56-71; Gilles Marsolais, "Mario Ruspoli" (pp. 137-138); "Regard sur la folie" (pp. 177-178), in L'Aventure du cinéma direct revisitée, op. cit.; Gilles Marsolais, "Regard sur la folie de Mario Ruspoli ", in L'aventure du direct, op. cit., pp. 235-237.

Ajoutons à ceci, deux articles dans Cinéma 63: Mario Ruspoli, "Remarques sur le cinéma direct, dit: “Cinéma-vérité`», Cinéma 63, mars 1963, $n^{\circ} 74$; "Les inconnus de la terre et Regard sur la folie", Cinéma 63, janvier 1963, n 72.

18 Guy Gauthier, Philippe Pilard, Simone Suchet, Le Documentaire passe au direct, op. cit., p. 71.

19 Les principaux protagonistes de ce mouvement sont, entre autres: Lucien Bonnafé, Georges Daumézon, Sven Follin, Louis Le Guillant, François Tosquelles, Yves Racine, Jean Oury, Jean Ayme. 
20 Je signale un article important qui va dans le sens des hypothèses développées ici. II est rédigé par Henri Ey, le médecin qui ouvrira la psychiatrie française sur le monde extérieur et qui manifestera pour la psychiatrie institutionnelle et ses partisans un intérêt soutenu et une sympathie sans faille: Henry Ey, "La psychiatrie devant le surréalisme", in L'évolution psychiatrique, vol. 13, 1948, pp. 3-52. Henry Ey y démontre la fécondité des liens entre psychiatrie et surréalisme, et plus précisément entre le fou et l'artiste surréaliste.

21 La situation excentrée et isolée de SaintAlban favorise en effet la rencontre de nombreux militants fuyant les régimes nazi et franquiste. le mouvement surréaliste et la psychiatrie institutionnelle 20 , une filiation qui s'accomplit principalement par la médiation de deux références théoriques (la psychanalyse et le marxisme), avant de revenir au film de Ruspoli qui actualise une approche cinématographique en harmonie avec l'esprit qui anime cette institution.

\section{Bonnafé et Tosquelles : entre surréalisme, psychanalyse et marxisme}

Situé à 1000 mètres d'altitude dans un château féodal sur le plateau sauvage du Gévaudan, l'hôpital de Saint-Alban accueille de 1939 à 1945, non seulement les malades des environs, mais également des intellectuels, des maquisards blessés, des résistants et des clandestins fuyant les régimes dictatoriaux ${ }^{21}$. L'hôpital est alors dirigé par le $\mathrm{D}^{\mathrm{r}}$ Pierre Balvet qui propose, dès 1936, des améliorations en vue d'humaniser l'asile. Il sera relayé à partir de 1942 par le $\mathrm{D}^{\mathrm{r}}$ Lucien Bonnafé, psychiatre dés-
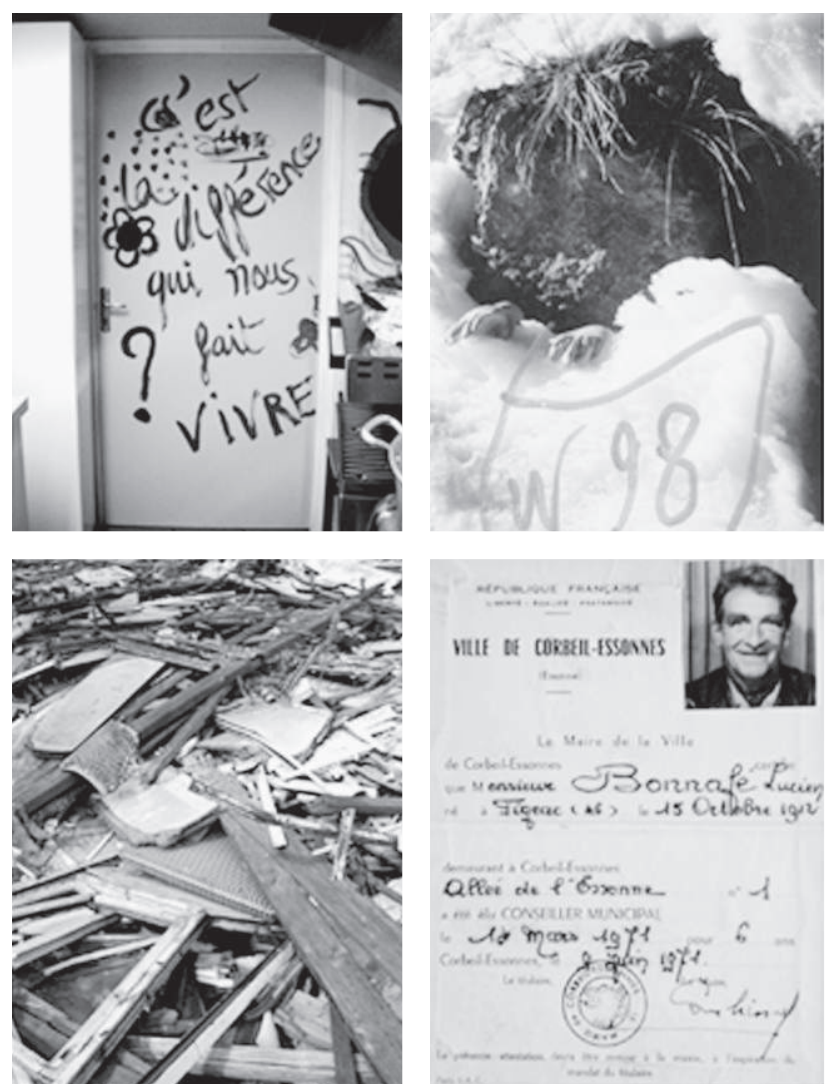
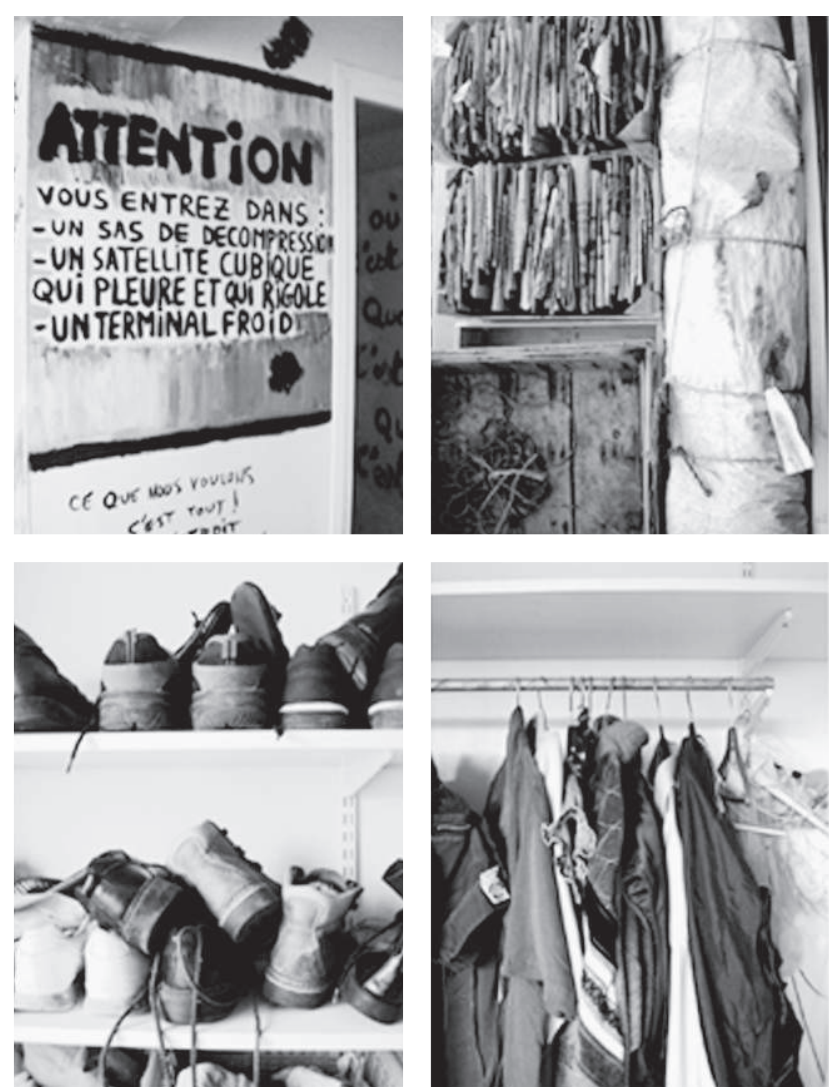
aliéniste de la première heure, anti-fasciste et syndicaliste convaincu et connu pour avoir mis en place la psychiatrie de secteur qui consiste à soigner le patient dans un périmètre géographique qui dépasse l'enceinte de la clinique et qui englobe notamment son domicile (et d'autres lieux de vie) (fig. 11). Avant la Guerre, Bonnafé participe au groupe surréaliste de Toulouse, se liant avec Max Ernst, Man Ray ou René Crevel au cours de ses séjours à Paris où il se rend régulièrement en tant que représentant de son cercle. Il s'implique également dans l'animation de ciné-clubs, d'éditions de poésie, tout en suivant les tentatives de réforme du front populaire qui prône l'élaboration de structures de soins extrahospitalières. C'est fort de cette double formation (marxiste et surréaliste) qu'il arrive à Saint-Alban où il va mettre en application ses réflexions sur les surdéterminations historiques et sociales de la folie, sur l'aberration de la politique d'internement systématique des malades mentaux et sur les affinités électives du délire avec la poésie.

Cette même année, Bonnafé fonde, aux côtés de Pierre Balvet, François Tosquelles et André Chaurand, la "Société du Gevaudan" qui pose les bases pratiques et théoriques de la psychiatrie institutionnelle qui se développera sous la double influence du marxisme et de la psychanalyse (fig. 12). Pendant l'occupation, cette Société se donnera pour mission de faire passer des ouvrages ou des passagers en zone libre, en mettant par exemple en contact des psychiatres séparés géographiquement. Durant cette période, Bonnafé reçoit Tristan Tzara, Paul Eluard (qui lègue à Saint-Alban une plate-forme d'édition clandestine, fig. 13), Georges Sadoul (alors agent de liaison de la Résistance 22), Jacques Matarasso (un surréaliste toulousain ami de longue de date) et Georges Canguilhem (qui s'investira dans les travaux collectifs et le soin aux patients). L'hôpital de Saint-Alban devient alors l'épicentre d'un bouillonnement intellectuel explorant, à la fois, les conséquences de l'oppression par l'occupant et la crise d'une société occidentale dévolue au capitalisme, à l'idéologie belliciste et au patriarcat. Quoique brève, la présence de Bonnafé en Lozère va marquer l'esprit des lieux et des personnes l'ayant côtoyé, à commencer par François Tosquelles qui va enseigner et amplifier à Saint-Alban, durant les deux décennies suivantes, une culture psychiatrique en rupture avec les normes établies dans ce milieu.

Euvrant énergiquement dans la lutte contre les totalitarismes, François Tosquelles est un psychiatre catalan, militant du P.O.U.M (mouvement trotskiste anti-stalinien), chef des Services psychiatriques de l'armée républicaine pendant la Guerre civile espagnole (1936-1939) et qui, suite à sa condamnation à mort par le régime franquiste, émigrera
22 A partir d'une petite indication contenue dans l'ouvrage d'Elisabeth Roudinesco, Histoire de la psychanalyse en France.2, j'ai tenté de trouver plus d'informations sur le passage de Georges Sadoul à Saint-Alban, sans grand succès (son journal, par exemple, couvre une période allant jusqu'en 1940). Voilà le passage en question: "A Saint-Alban se retrouvent pêle-mêle des résistants, des fous et des thérapeutes. Paul Eluard et Georges Sadoul s'y cachent et lisent les textes des aliénés." (Elisabeth Roudinesco, Histoire de la psychanalyse en France. 2 (1925-1985), Paris, Fayard, 1994 , p. 204). Seule une interview de la fille de Bonnafé, Marie Bonnafé (née en 1935), permet de confirmer sa présence parmi les invités du médecin-psychiatre qui avait l'habitude d'accueillir chez lui ses visiteurs. Cet entretien permet de situer la rencontre entre les deux hommes entre janvier 1943 et l'été 1944 (se rapporter au document audio mis en ligne sur le site Internet consacré à Lucien Bonnafé: www.lire-lucien-bonnafe.org/index.php?cat= gevaudan). Voir aussi: François Dosse, Gilles Deleuze et Félix Guattari. Biographie croisée, Paris, La Découverte, 2007, p. 56. Notons que, curieusement, Sadoul, dans son commentaire du film, n'évoque pas du tout son passage à Saint-Alban, alors qu'il s'est toujours épanché facilement sur ses activités de résistant. Georges Sadoul, "Les progrès du cinémavérité", in Lettres françaises, 14 décembre 1961. Georges Sadoul, "A Lyon, les caméras vivantes ont rencontré le cinéma-vérité ", in Lettres françaises, $n^{\circ}$ 970, mars 1963. 
23 Alors que Tosquelles est un médecin-chef fort réputé dans son pays, il doit recommencer sa formation depuis le début en tant qu'infirmier-adjoint, le France ne reconnaissant pas les diplômes étrangers. De 1946 à 1953, il franchira tous les échelons de la hiérarchie jusqu'à sa nomination, en 1953, au poste de médecin directeur de Saint-Alban. Sur l'œuvre, la pensée et la vie de François Tosquelles, voir: Patrick Faugeras, L'ombre portée de François Tosquelles, Ramonville Saint-Agne, Eres, 2007. Ses écrits et entretiens abondent également en informations utiles: François Tosquelles, L'enseignement de la folie, Paris, Privat, 1992; François Tosquelles, "Une politique de la folie ", in Chimères, automne 1991, n 13, pp. 66-81 (cet article contient la transcription intégrale du documentaire François Tosquelles, une politique de la folie, réalisé par François Pain, Danielle Sivadon et Jean-Claude Polack, 1990); Jean Oury, Félix Guattari, François Tosquelles, Pratique de l'institutionnel et politique, Vigneux, Matrice Editions, 1985; François Tosquelles, "Quelques aperçus sur l'histoire de la psychothérapie", in L'information psychiatrique, juin 1964, $\mathrm{n}^{\circ}$ 6, pp. 401-416; François Tosquelles, "La Société vécue par les malades psychiques", in Esprit, décembre 1952, pp. 897-904.

24 Septfonds est l'un de ces lieux concentrationnaires qui permet à l'administration française de cantonner 450000 réfugiés espagnols. Après avoir vécu les conditions extrêmes d'une guerre civile, Tosquelles, à nouveau confronté à la famine, aux maladies, aux épidémies, aux suicides de ses compagnons d'infortune, crée un service de soins psychiatriques basé sur la solidarité et la contestation de I'ordre établi. François Tosquelles, "Une politique de la folie", id., p. 73.

25 Jacques Lacan, De la psychose paranoïaque dans ses rapports avec la personnalité [1932], Paris, Seuil, 1975. Arrivé à Saint-Alban, Tosquelles fera lire la thèse de Lacan à tout le personnel, y compris aux infirmiers. Tosquelles s'initie à la psychanalyse d'abord via son maître, le Professeur Emilio Mira I Lopez qui diffuse la doctrine freudienne en Espagne, puis par ses contacts avec des psychanalystes hongrois et autrichiens ayant fui le nazisme. II suivra luimême, dès 1931, et malgré les obstacles linguistiques, une psychanalyse avec un analyste juif réfugié à Barcelone, Sandor Reminger. (François Tosquelles, "Une politique de la folie", id., p. 70).

26 II écrit à ce propos: "C'est en mettant en valeur l'opérativité de l'association libre des

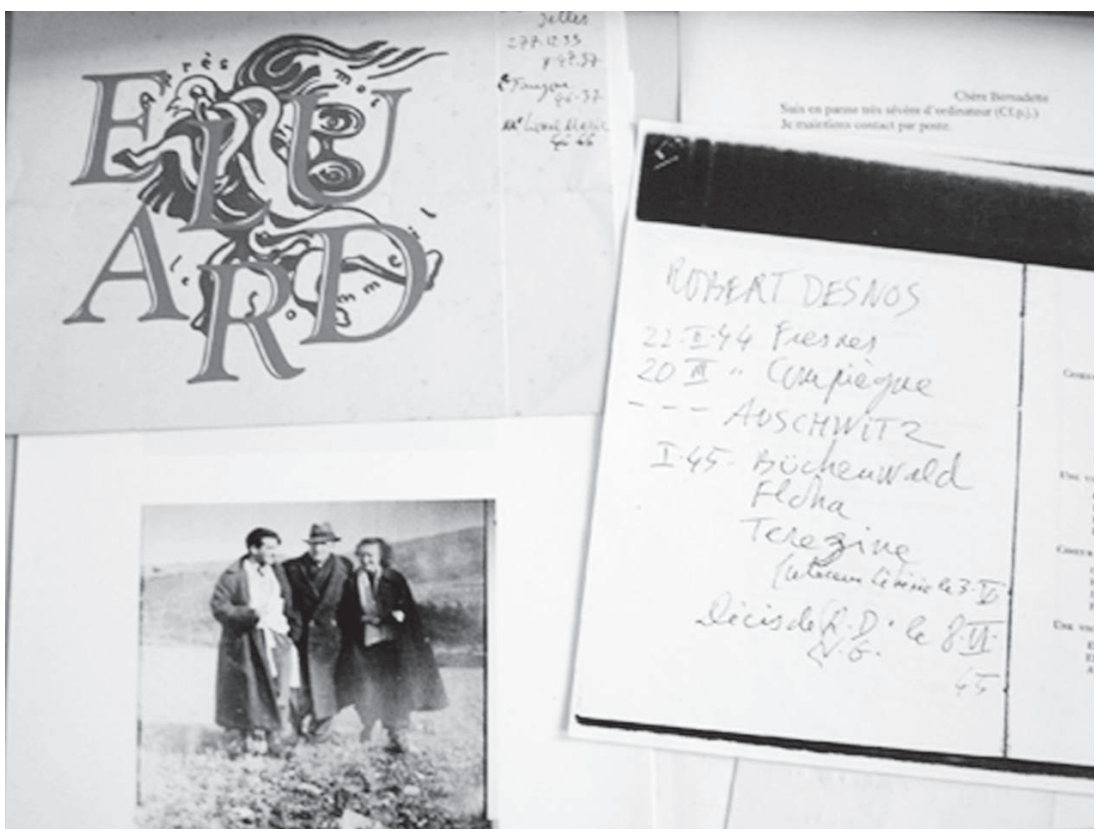

12

en France 23. Il est invité à travailler à Saint-Alban en janvier 1940, après avoir séjourné dans le camp de concentration français de Septfonds destiné aux réfugiés indésirables 24 . Figure phare de la psychiatrie institutionnelle, Tosquelles (fig. 14) amène avec lui son activisme politique (sa sensibilité marxiste), son héritage culturel (dont le surréalisme), sa passion pour la psychanalyse (il fait imprimer par l'association des patients, appelée le Club des malades, la thèse de Jacques Lacan sur la psychose ${ }^{25}$ ), ses modèles théoriques (la phénoménologie et la psychiatrie allemandes léguées par son maitre et compatriote, le psychiatre Emilio Mira I Lopez), enrichissant ainsi un terrain déà imprégné par ces influences. Du surréalisme, il retient son goût pour l'imprévu et l'inattendu, son éloge de la liberté humaine $\mathbf{2 6}$, ainsi que ses liens étroits avec la psychanalyse et le marxisme perçus comme des ensembles théoriques qui se rendent à l'évidence des processus dialectiques et contradictoires de l'âme humaine ${ }^{27}$. Au cœur de cet agrégat référentiel, la question du langage fonde une conception de la folie comme étant radicalement et immédiatement branchée sur l'inconscient.

Travaillant en excellente intelligence avec Bonnafé, Tosquelles propose de traiter la psychose en se référant à la pensée freudo-lacanienne sur l'aliénation individuelle et à l'analyse marxiste de l'aliénation so- 


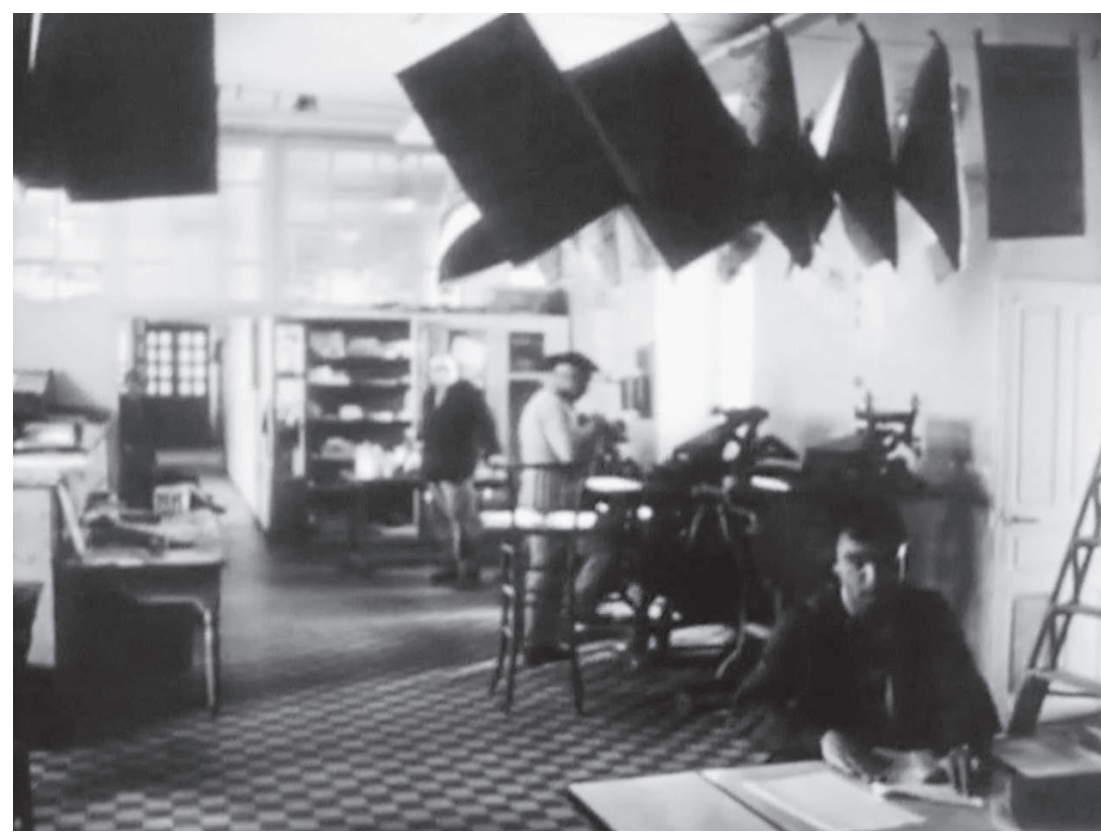

13

ciale. Car si, comme le suggère Bonnafé, «la psychanalyse met en évidence les difficultés d'évolution des rapports de l'être dans la famille comme facteur de désadaptation sociale, [...] la méthode historique [préconisée par le marxisme] nous apporte, sur les problèmes de la famille, les possibilités d'une compréhension scientifique» 28 . Il s'agit dès lors de déconstruire les mythologies attachées à la folie, en soulignant son caractère historique et social (la psychanalyse consignant une crise généralisée du primat paternel dans la société occidentale), et en libérant l'être

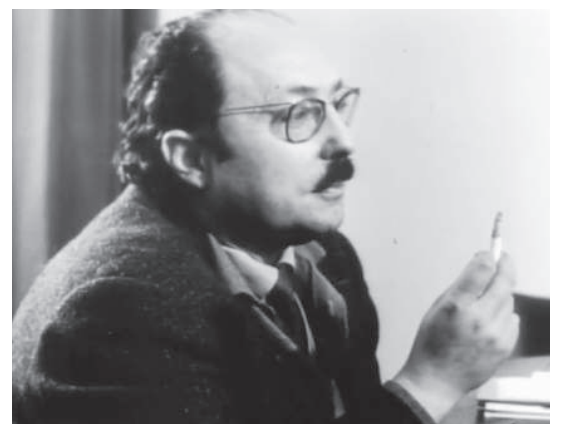

14 idées, au cours de la prétendue cure thérapeutique analytique, que la liberté elle-même peut jouer un rôle positif au cours de la pensée qui se veut logique. Les apparences absurdes, et à vrai dire surréalistes, de l'association libre des idées constituent le vrai ‘sur-plus) du réel abordable par les hommes." (François Tosquelles, L'enseignement de la folie, op. cit., p. 92).

27 "Les surréalistes n'essayent pas de côtoyer seulement les démarches freudiennes, mais aussi le marxisme, peut-être en accentuant le déterminisme historique et social." (id.). Ailleurs, Tosquelles rapproche les "couples pulsionnels" (passif/actif; sadisme/masochisme, etc.) de Freud, qui "jouent ensemble, sans pour autant s'annuler", à la "coexistence de la ‘ thèse) et de l'‘ antithèse " " chez Hegel et Marx (François Tosquelles dans Pratique de l'institutionnel et politique, op. cit., p. 109). Au travers de ses écrits et entretiens, il apparaît que Tosquelles se réfère principalement à trois protagonistes du surréalisme: André Breton, Salvador Dalí et Louis Aragon. Du premier, il signale sa lecture romantique de l'Interprétation des rêves de Freud et son modèle de subjectivité d'orientation psychopathologique. (François Tosquelles, L'enseignement de la folie, op. cit., p. 92). II commente notamment la rencontre Breton et Freud en 1922; du deuxième, il met en évidence sa fidélité à une culture catalane ultra scientifique et sa formulation d'une théorie réaliste de la psyché qu'il configure dans les traits de sa méthode paranoïa-critique (dont il sera question plus loin). (id.); du dernier, qu'il nomme "la dentellière de la parole", il rappelle une rencontre émouvante qui s'est tissée autour d'un goût commun pour la poésie, la musicalité de la langue et les jeux de langage. (François Tosquelles, "Une politique de la folie", op. cit., pp. 75-76).

28 Lucien Bonnafé, "Essai d'interprétation du fait psychiatrique selon la méthode historique de K. Marx et F. Engels (Histoire d'un mythe)", in L'évolution psychiatrique, vol. 13, 1948, $\mathrm{n}^{\circ}$ spécial, p. 94. 
29 Bonnafé affirme: "Dans un ordre social nouveau fondé sur une organisation non oppres sive des moyens de production, sur la suppression des antagonismes de classe, avec une structure familiale basée sur l'égalité réelle des sexes, donc non paternaliste, la nature de la folie ne saurait rester la même, ni la connaissance psychiatrique. (id., p. 102). [...] Les solutions des problèmes de la folie, conclut Bonnafé, dépendent de la liquidation de l'aliénation humaine, c'est-à-dire de la libération de I'homme vis-à-vis de ses moyens de productions et de ses produits. La psychiatrie ne gouverne pas cette libération, elle l'entrave Iorsqu'elle prétend gouverner. Ses aspirations à la libération concrète de certains hommes que l'on appelle les fous obéissent aux lois qui régissent l'avènement du règne de la liberté [...]" (p. 105).

30 Tosquelles répète tout au long de ses écrits combien chaque patient se rattache à une multitude d'institutions dont la cartographie complexe est à traiter au même titre que le malade. L'hôpital psychiatrique apparaît, selon lui, comme "un ensemble de plusieurs institutions articulées et intégrées [...]. La liberté humaine se mesure en quelque sorte par l'existence de plusieurs institutions auxquelles chacun peut dès lors participer. Cette pluralité d'institutions est pour moi fondamentale. C'est que ce qui à mon avis rend possible le changement que chacun peut vivre, lorsqu'il passe, de luimême, d'une logique de l'appartenance (à n'importe quel groupe) à une logique de participation. "(François Tosquelles, L'enseignement de la folie, op. cit., p. 235).

31 François Tosquelles dans Pratique de l'institutionnel et politique, op. cit., p. 92.

32 Mario Ruspoli, "Remarques sur le cinéma direct, dit: ‘Cinéma-vérité`", op. cit., p. 143.

$33 / d$. , p. 144. On peut à ce propos tirer un parallèle entre les réunions hebdomadaires des psychiatres de Saint-Alban qui discutent de leur propre travail réalisé durant la semaine (comme le montre une des séquences du film), et la volonté de Ruspoli de créer une cohésion autour d'un même film, de sorte que chacun des intervenants fasse synergie avec l'ensemble.

34 /d., pp. 144-145.

35 /d., p. 145.

36 D'après nos recherches, et avec l'aide précieuse de Séverine Graff que je remercie ici, il humain du joug du familialisme, du patriarcat et de l'ordre dominant, de sorte à lui permettre de recréer ailleurs un nouvel agencement subjectif et relationnel libéré de ces entraves 29.

La psychiatrie institutionnelle s'emploie donc, grâce à différentes institutions, à lutter contre les systèmes d'oppression matériels et imaginaires, en s'appuyant sur le versant sain du malade appelé à prendre une part active dans la collectivité, et partant, dans sa cure ${ }^{\mathbf{3 0}}$. Amenés à élaborer les ressources et les conditions d'une psychothérapie d'orientation psychanalytique, la communauté et les individus qui la composent deviennent l'objet de soins qui ne dépendent plus des conventionnelles séances sur le divan, mais qui laissent les personnes circuler librement dans des espaces non clôturés prêts à accueillir des opérations psychiques de toute nature. L'hôpital, avec son organisation interne et ses prolongements alentours, devient alors le théâtre d'échanges conscients et inconscients sur lesquels s'applique une analyse médicale ouverte et mobile s'adaptant elle-même aux flux d'énergie libidinale dégagés par ces interactions - ce que Tosquelles nomme les «libérations psychodramatiques» $\mathbf{3 1}$ - de manière à favoriser autant que possible l'émergence de la créativité de la parole et des rencontres qui lui sont attachées. Tous les moyens de communication et de création sont par conséquent encouragés, comme le prouve la mise sur pied, dès 1950, d'un journal interne de libre expression, Le Trait d'Union qui rapporte témoignages, textes en prose ou poétiques, revendications individuelles, faits d'actualités, etc., et dont le documentaire fait état dans une séquence (rédigé, imprimé et vendu au sein de l'hôpital, ce journal paraîtra jusqu'en 1981), (fig. 15).

\section{Un cinéma/une psychothérapie de la parole et de l'audition}

Réalisé sur les bases du cinéma direct, «un cinéma plus libre, un cinéma de contact»32, Regard sur la folie peut être considéré comme un équivalent cinématographique de cette thérapie reposant sur le paradigme désaliénant du corps, de la parole, de la pensée. Non seulement le cinéma direct, tel que pratiqué par Ruspoli, vise à redonner une parole aux gens ordinaires voire méprisés, mais il cherche aussi à s'inscrire dans l'horizontalité des rapports humains, en faisait fi des jeux de pouvoir qui la plupart du temps les régulent. Cette approche souple et collégiale, perceptible dans la manière dont les techniciens se mêlent aux personnes filmées et prennent part aux discussions ou activités, rappelle évidemment certains principes qui guident la psychothérapie institutionnelle, à commencer par celui du travail en équipe. Ruspoli conçoit en effet cette «nouvelle manière de travailler [...] ‘ comme un 
seul homme»» comme un réponse adressée au lourd carcan imposé par les codes et le dispositif technique du cinéma classique dont il s'agit de «faire table rase» $\mathbf{3 3}$. L'ambition nourrie par Ruspoli consiste, en effet, à :

"Pouvoir filmer autour de soi, immédiatement, avec le son à l'appui, pouvoir capter les événements, la vie, les hommes et leurs rapports entre eux, s'inscrire dans le cadre de l'existence par le Cinéma, pouvoir en transcrire ensuite efficacement le témoignage. »34

Ce cinéma de proximité qui fait dissidence avec une tradition qui le précède, qui déconstruit les "clichés sociaux et culturels» 35 et qui respecte l'identité des êtres humains et des situations, fait évidemment écho à la démarche avant-gardiste d'une psychiatrie dévolue à une prise en charge empathique du fou. La rencontre à Saint-Alban, en 1961, entre Ruspoli et Tosquelles, ne se résume pas seulement à une conjonction humaine très vraisemblablement réalisée par l'intermédiaire de l'oncle du cinéaste, Gilbert de Chambrun ${ }^{36}$. Elle se noue aussi autour de cette aspiration commune à remettre en cause, dans leurs champs respectifs - le cinéma, la psychiatrie - pratiques et conceptions dominantes, de sorte à fluidifier les liens entre individus, institutions et société. Fluidification de la prise

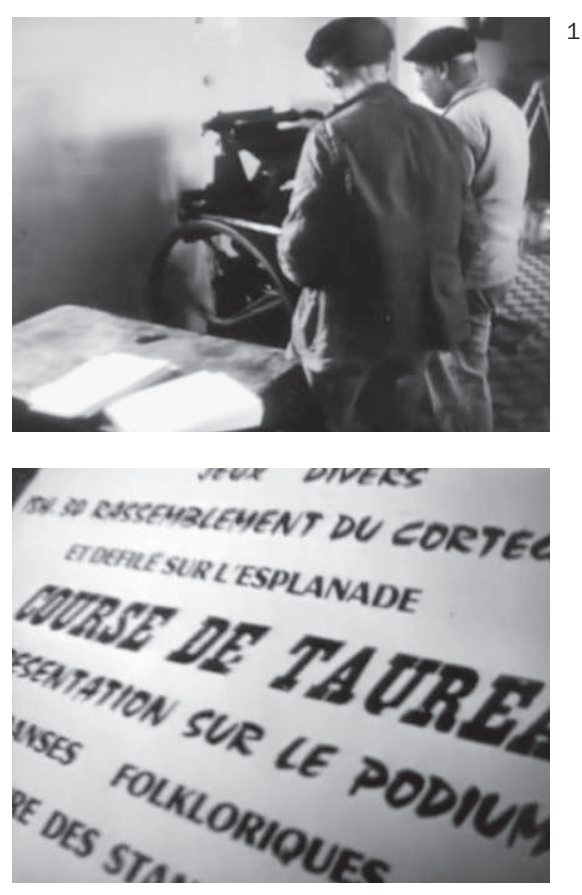

semblerait que Tosquelles et Ruspoli se soient rencontrés à Marjevols, petite ville proche de Saint-Alban où Ruspoli a passé son enfance chez sa mère, au château de Marvejols (et où il résidera avec son équipe le temps du tournage du film). C'est très certainement par l'intermédiaire de son oncle, Gilbert de Chambrun, maire de la ville et ami de Tosquelles, que Ruspoli et le psychiatre catalan sont entrés en contact. Tosquelles se rend en effet régulièrement à Marjevols pour travailler au Clos-du-Nid, une institution pour enfants arriérés profonds qu'il a fondée avec l'abbé Oziol en 1955. La mention du nom de Gilbert de Chambrun dans l'entretien que Sivadon et Polack font de Tosquelles pour leur film François Tosquelles, Une politique de la folie, atteste d'un lien amical et politique entre l'oncle de Ruspoli et Tosquelles. Et pour cause: Gilbert de Chambrun est un homme politique français, adhérant au Parti Communiste, très proche des Républicains espagnols et qui sera nommé durant la Guerre chef de la Résistance en Lozère. Comme d'autres amis français, il soutiendra Tosquelles contre les brimades dont il est victime dès son arrivée en France en tant qu'étranger, espagnol et homme de gauche. Un article de la revue Cinéma 63 permet de préciser un point concernant la genèse du film: installé à Marjevols avec ses collaborateurs (dont Pierre Lhomme et Michel Brault), Ruspoli est disposé à filmer autour de lui tout ce qui lui semble intéressant. II ramènera de ce séjour quarante heures de rushes dont seront tirés trois documentaires: Les Inconnus de la terre (Mario Ruspoli, France, 1962), Regard sur la folie et La Fête prisonnière (les deux derniers constituant les volets d'un même film), tous datés de 1961. C'est en tournant Les Inconnus de la Terre, un documentaire sur la vie paysanne en Lozère, "enquête anthropologique" menée en étroite collaboration avec Roger Gentis et François Tosquelles, qu'il aura l'idée - très certainement suggérée par Tosquelles lui-même - de donner la parole à des paysans devenus fous soignés à Saint-Alban (Raymond Bellour, "Ruspoli au pays des hommes", in Cinéma 63, mai 1963, n 76 , pp. 25-27). Un article de L'Express contredit toutefois cette version des faits, sans que nous puissions ici trancher définitivement sur le lien de causalité existant entre les tournages des Inconnus de la terre et Regard sur la folie: "C'est un médecin de Saint-Alban qui a eu l'idée de conduire l'équipe de Ruspoli chez des paysans à qui il avait confié un de ses malades, un enfant mongolique." (L'Express, 22 février 1962, p. 27). 
37 Toutes les études sur le cinéma direct, ainsi que ses instigateurs eux-mêmes, mettent en exergue l'importance accordée à la parole et à sa captation grâce aux nouveaux dispositifs d'enregistrement du son. En voici un exemple: "Convaincu que le son, enfin synchrone avec l'image, est un moyen d'investigation efficace, [Mario Ruspoli] pense que les cinéastes peuvent désormais donner la parole à ceux qu'on appelle de façon méprisante les 'gens ordinaires ', condamnés au mutisme par le commentaire omniscient." (Guy Gauthier, Philippe Pilard, Simone Suchet, Le Documentaire passe au direct, op. cit., p. 57). Voir aussi, l'article de Michel Serceau, "L'avènement du cinéma direct et la métamorphose de l'approche documentaire", in CinémAction, $n^{\circ} 41$, op. cit., pp. 78-87. Le constat de la primauté de la parole dans le cinéma direct doit être envisagé dans un contexte plus large englobant une réflexion sur la réciprocité des influences entre appareils de prise de vue/son et esthétique, comme le démontrent les travaux de Séverine Graff qui, à rebours de I'historiographie classique, déconstruisent précisément les rapports mécaniques de cause à effet entre innovations technologiques et apparition d'un nouveau langage cinématographique. Séverine Graff, "L'influence des technologies dans l'émergence du cinéma-vérité. Stefan Kudelski et l'invention du magnétophone Nagra III", in Kino CH/Cinéma $\mathrm{CH}$, Rezeption, Ästhetik, Geschichte/Réception, esthétique, histoire, Alain Boillat, Philipp Brunner, Barbara Flückiger (Hg./éd.), Marburg, Schüren, 2008, pp. 233-245.

38 Saint-Alban distribuait des cartes d'adhérents, sur le modèle du parti communiste. Voir fig. 16: carte d'adhérente de Dolorès Ruspoli (Grassian).

39 Mario Ruspoli, "Remarques sur le cinéma direct, dit: ‘Cinéma-vérité`», op. cit., p. 145. de son/de vue d'un côté37, fluidification des rapports psycho-sociaux de l'autre, il s'agit dans les deux cas d'instaurer les conditions favorables à un affranchissement de la parole reçue dans une attitude d'éveil et de non jugement.

Le cinéma direct et la psychiatrie institutionnelle partagent en effet un certain nombre de caractéristiques comparables, toutes subsumées par une forme d'engagement éthique (voire politique) au sein d'une communauté animée par un esprit d'entraide et de gestion collective des problèmes $\mathbf{3 8}$ : une volonté de renouveler le cadre de leurs pratiques; l'implication de l'équipe enquêtrice (les opérateurs de filmage et le personnel soignant); l'écoute attentive des personnes filmées/soignées; l'importance accordée à la parole comme lieu d'une "vérité" à restituer sans la diriger ou la trahir; le respect d'une non-hiérarchie entre filmants/filmés, soignants/soignés; le souci de préserver la créativité des individus pris à parti. Ce n'est donc pas un hasard si Mario Ruspoli luimême tire un parallèle entre sa démarche et celle du psychiatre:

"C'est ainsi que nous avons cherché à remplacer l'interview directe', qui est le pain quotidien de la télévision, par une attitude d'expectative, de présence non interrogative, modelée sur celle des psychiatres. [...] Nous ne préparons jamais de questionnaire, et abordons le problème de la prise de vue sans jamais présager de ce qu’elle pourra révéler.»39

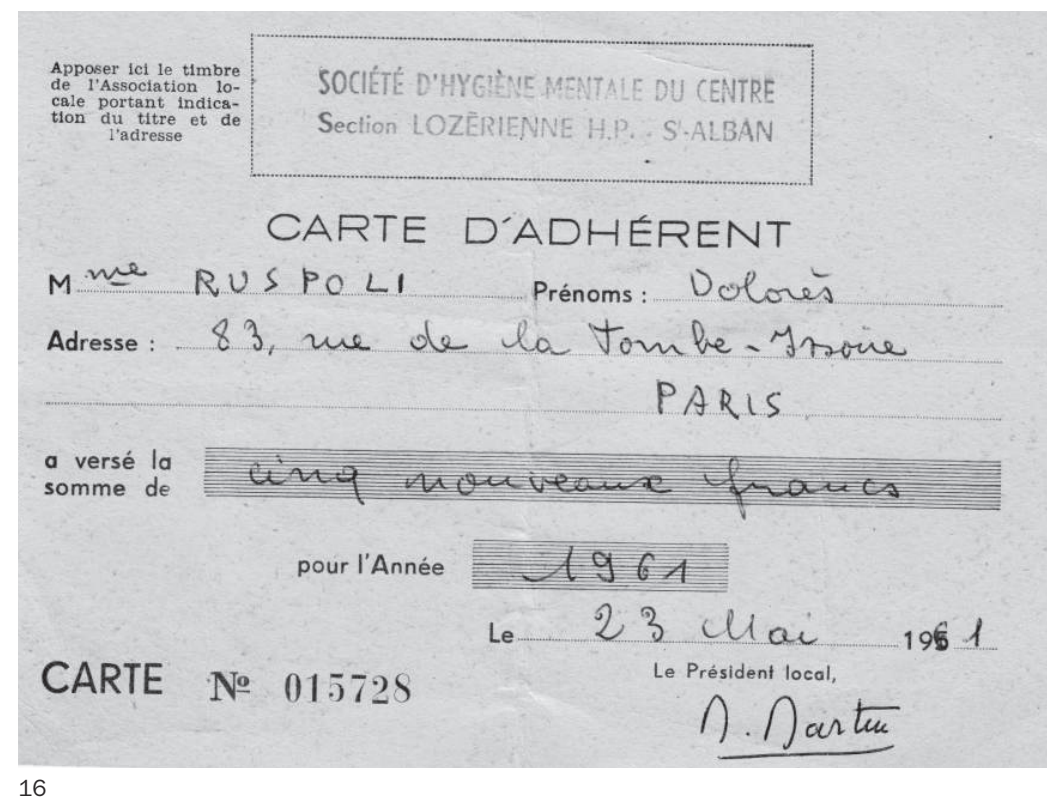


Très soucieuse d'éviter toute forme d'intrusion - Ruspoli parle du risque de "violer» $\mathbf{4 0}$ ou de "traumatiser» $\mathbf{4 1}$ les gens filmés - l'équipe doit se fondre dans l'environnement choisi, employant si nécessaire la complicité d'une personne qui «se trouve dans la même orbite de recherche que nous» et qui "devient un précieux collaborateur, un irremplaçable moyen de pénétration psychologique dans le milieu social auquel il appartient». Etayant à nouveau sa réflexion, dans son rapport à l'UNESCO, sur la métaphore de l'observation psychologique, Ruspoli met en exergue la nécessité pour l'équipe de tournage de s'effacer autant que possible pour laisser libre court aux événements filmés :

"Se faire oublier, appartenir au paysage, se confondre avec la foule, est une attitude fondamentale pour le cinéaste qui cherche à approcher le réel. Il doit abandonner toute personnalité apparente, tout détail qui le ferait remarquer. Les techniciens du 〈cinéma direct) sont aussi des psychologues et leur attitude d'reffacement` nécessite une profonde connaissance du comportement humain lié à la passion de son étude. [...] Cet art du mimétisme s'apprend et devient une seconde nature. » $\mathbf{4 2}$

Même si, dans Regard sur la folie, cet idéal n'est pas toujours atteint - certains malades désignant l'instance de filmage située hors-champ, à l'instar de $\mathrm{M}^{\mathrm{me}}$ Blanche qui dit reconnaître une ou deux personnes lors de sa conversation avec un psychiatre -, le but pour Ruspoli consiste à créer un espace ouvert pour qu'advienne une réalité non préméditée. Ce mode de filmage dépend beaucoup du concept de "caméraman libre» $\mathbf{4 3}$ qui s'investit complètement dans le processus, à l'image du médecin qui fait presque corps avec sa patiente, tout en lui permettant de s'exprimer librement. Car, pour Ruspoli, «c'est aussi une question psychique : être ou ne pas être <engagé > en tant que talent libre, dans ce type de tournage de 〈participation〉 directe aux événements» $\mathbf{4 4}$. A la métaphore psychologique, se joint la métaphore musicale, Ruspoli comparant le métier des opérateurs du cinéma direct à celui d'un "petit orchestre de jazz, habitué à improviser collectivement» $\mathbf{4 5}$. Travail d'équipe, éthique personnelle et professionnelle, capacité d'adaptation à l'inattendu et créativité permanente forment ainsi une base commune aux «cinéastes-vérité » et aux psychiatres de Saint-Alban.

Au sein de ce dispositif de capture du spontané et de l'imprévisible, la parole occupe, comme dans le dispositif de la cure psychanalytique, une place structurante et structurale. Ainsi, Ruspoli écrit-il :

"Chez certains malades mentaux, momentanément figés dans des structures rigides, le langage s'exprime de manière très différente. Les barrages n'existent pas au même niveau et c'est au psychiatre de
40 "Là encore, jamais le cinéaste ne doit tâcher de ‘violer, les gens". (Mario Ruspoli, "Remarques sur le cinéma direct, dit: ‘Cinémavérité >", id., p. 146).

41 "Dans ce désir de ne pas créer de traumatisme chez ceux que nous voulons filmer, par la vue des opérations techniques: la charge et la décharge de la pellicule, le branchement des câbles électriques, l'installation des éclairages, nous arrivons dans un lieu de tournage déjà prêts à filmer, et nous tâchons dans la mesure du possible, d'y être avant tout le monde, de manière à ne pas causer de surprise par une arrivée intempestive". (Mario Ruspoli, "Remarques sur le cinéma direct, dit: ‘Cinémavérité> ", id., p. 147).

42 Mario Ruspoli, Pour un nouveau cinéma dans les pays en voie de développement: le groupe synchrone cinématographique léger, Paris, Unesco, 1963 (document disponible sur www.decadrages.ch) et cité par Guy Gauthier, Le documentaire, un autre cinéma, op. cit., p. 118.

43 Mario Ruspoli, "Remarques sur le cinéma direct, dit: «Cinéma-vérité`», op. cit., p. 149.

44 lbid.

$45 \mathrm{lbid}$. 


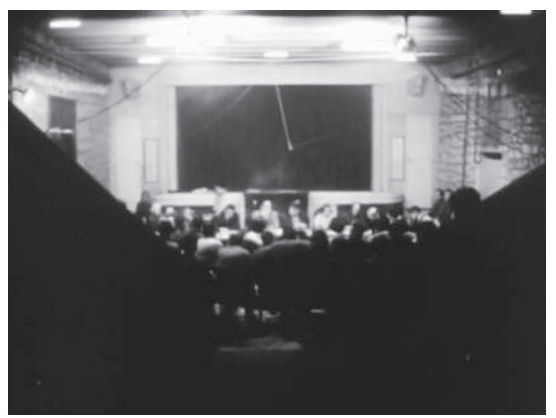

17

46 Ibid.

47 François Tosquelles, L'enseignement de la folie, op. cit., pp. 122-123.

48 François Tosquelles, "Une politique de la folie", op. cit., p. 78.

49 Tosquelles, dans le documentaire qui lui a été consacré, évoque cette séquence en récitant notamment ce poème (id.).

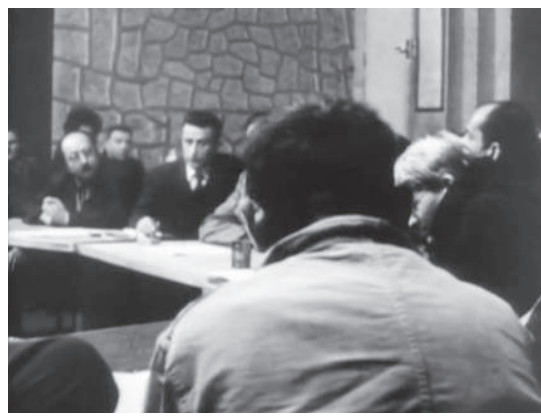

18

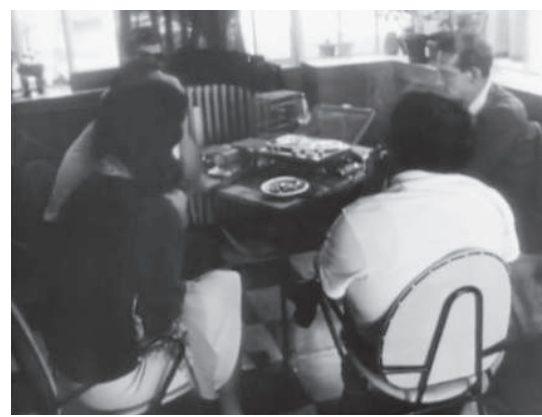

19 faire l'effort pour le comprendre, pour s'éclairer lui-même sur la pensée du malade. Nous sommes venus à penser que la recherche d'une certaine 'vérité> était étroitement liée à la recherche du langage, et que le langage n'avait rien d'universel, mais était un fil ténu et subjectif, sujet à toutes sortes d'oscillations.» 46

Cinéma de la parole et de l'audition, comment ne pas trouver dans la psychothérapie institutionnelle et dans les fous dont elle perçoit attentivement les délires, une cousine approche de l'humain? L'attention expectante portée par Ruspoli et Tosquelles sur la parole des personnes filmées/soignées opère sur une modalité analogue que l'on peut mettre en parallèle avec l'écoute flottante de la psychanalyse censée favoriser la libre association des idées de l'analysant comme de l'analysé. Tosquelles, qui garde toujours en arrière-fond le modèle surréaliste du collage d'idées et d'images hétérogènes, explicite ainsi une méthode fondée sur le vagabondage des corps et des esprits au sein de l'hôpital, et qui n'est pas sans rapport avec celle préconisée par Ruspoli:

"La loi [des associations libres] s'établit en marchant et en rencontrant les autres, en se laissant aller à la fluidité des discours dé-socialisés, c'està-dire hors des formes précises qui marquent le point de départ de la rencontre où le discours circule.» $\mathbf{4 7}$

Pour expliciter cette loi, Tosquelles prend l'exemple des réunions du Club qui rassemble, chaque samedi, l'ensemble des membres de la communauté hospitalière dans le but, notamment, de discuter les textes de patients publiés dans le journal, le Trait d'Union, à l'instar du poème la «La nature crie» (une réunion de ce genre fait l'objet d'une longue séquence du film, fig. 17):

"Le Club était un lieu où les vagabonds pouvaient se retrouver, le lieu d'une pratique et d'une théorisation du vagabondage, de l'éclatement, de la déconstruction-reconstruction. [...] Sans ce vagabondage [...], on ne saurait parler de Droits de l'Homme. Le premier droit de l'homme est le droit au vagabondage. $\mathbf{4 8}$

L'environnement dont il est question se prête ainsi à la prise de parole non concertée, comme le patient (le "poète-fou» évoqué plus haut) qui récite à l'assemblée son poème La victoire de Samothrace, cette scène du film - que Tosquelles donne comme exemple d'«art brut» pris sur le vif - contenant des images cadrant, dans le même plan, le patient de dos et les médecins, face à lui, concentrés dans leur écoute (fig. 18) 49. Le dispositif de prise de vue et de son dont disposent les techniciens du cinéma direct est on ne peut mieux adapté à l'enregistrement de cette "institution en acte» qui se manifeste à travers des "rencontres à l'écoute - dans ces groupes de parole ou d'échanges» qui fournissent, 
pour Tosquelles, «la matière première indispensable à la psychothérapie institutionnelle» $\mathbf{5 0}$.

Une séquence "métadiscursive», croisant mise en abyme cinématographique et autocritique des psychiatres, désigne le soin apporté à cette double audition filmique/thérapeutique, à savoir celle qui présente les médecins et les techniciens écoutant les propos d'un patient autour d'un appareil à bande magnétique (fig. 19). Commentant le déchirement entre "oreille psychiatrique» et "oreille publique», selon les termes de Tosquelles, les médecins s’interrogent en effet sur le danger de réaliser un film de propagande qui réduirait l'ambiguïté fondamentale de la folie et de son traitement $\mathbf{5 1}$. En filigrane, apparaît la nécessité de préserver autant que possible le langage de la folie, de manière à ce que l'image qui en est donnée conserve son ouverture vers une série de significations infinie. La médiation par le dispositif cinématographique offre ainsi l'occasion aux psychiatres et aux opérateurs de réfléchir ensemble (donc comme "un seul homme») aux questions techniques posées par la captation visuelle et auditive de ce langage de la folie $\mathbf{5 2}$.

La version finale du film semble avoir apporté une solution à ce problème puisque la parole enregistrée envisage réellement l'image comme «un matériau vivant et multiple. Elle ne l'atrophie pas mais la prolonge et en donne quantité d'interprétations »53. La manière dont la parole investit l'image ruspolienne peut à cette occasion être éclairée à la lumière de la notion de "parole-relais» mise en évidence par Roland Barthes pour expliciter la manière dont certaines images (notamment cinématographiques) entrent dans un rapport de complémentarité avec la parole (ou le dialogue), sans assumer pour autant la «fonction simple d'élucidation». La parole-relais, contrairement à la "parole-ancrage» qui cherche à résorber la polysémie de l'image, fait ainsi «véritablement avancer l'action en disposant, dans la suite des messages, des sens qui ne se trouvent pas dans l'image» $\mathbf{5 4}$. Ni répressive, ni contrôlante, ni assujettie, la parole (celle des sujets parlants comme celle du texte filmique) acquiert ici une dimension poétique qui dépasse la simple qualité de déchiffrement, car, comme le rappelle Tosquelles, non sans malice, "le langage ne sert pas seulement à la formulation de messages et à leur décodage : ça c'est bon pour les télégraphistes» $\mathbf{5 5}$.

\section{La "vérité " de la parole du fou}

Dans Regard sur la folie, plus que l'image du fou, c'est surtout sa parole qui intéresse et fascine à part égale les opérateurs et les psychiatres, à l'affût qu'ils sont d'une polyphonie discursive riche en potentialités expressives et interprétatives (fig. 20) ${ }^{56}$. Car le psychotique donne à voir
50 François Tosquelles dans Pratique de l'institutionnel et politique, op. cit., pp. 124-125.

51 Marsolais, corrigeant ses propos de 1971 qui déploraient, à tort, l'absence d'intérêt du film pour les moyens thérapeutiques utilisés à Saint-Alban, écrit à propos de cette séquence: "En fait, Mario Ruspoli s'intéresse à différents aspects thérapeutiques de l'hôpital, incluant même un bref retour critique des médecins sur leur travail, insistant que la conscience qu'ils ont d'être filmés, mais ces ‘regards ‘ en éventail restent allusifs qui portent moins sur les traitements et les résultats obtenus que sur une certaine conception de la folie". Ce que Marsolais considère comme étant une faiblesse du film, constitue précisément sa force, Ruspoli captant au plus près de sa vérité un phénomène complexe - la psychose - qui guide toute la réflexion théorique et l'approche pratique de la psychiatrie institutionnelle (Gilles Marsolais, L'Aventure du cinéma direct revisitée, op. cit., p. 177).

52 Ruspoli précise: "Cette recherche passionnée du langage nous a orientés tout d'abord vers le problème technique de l'écoute." (Mario Ruspoli, "Remarques sur le cinéma direct, dit: "Cinéma-vérité'", op. cit., p. 145).

53 Mouloud Boukala, Le dispositif cinématographique, un processus pour [re]penser l'anthropologie, op. cit., p. 112.

54 Roland Barthes, "Rhétorique de l'image", in L'obvie et l'obtus. Essais critiques III, Paris, Seuil, 1982, p. 33.

55 François Tosquelles, L'enseignement de la folie, op. cit., p. 243.

56 "Tout discours entendu devient en fait polyphonique, au-delà de l'indicatif des messages conscients, ça traîne partout de l'inconscient: chaque mot, chaque phrase traîne du passé méconnu: une longue histoire, qui s'étale ainsi et se faufile pendant des siècles entre des groupes humains successifs, sautillants. Ça arrive ainsi toujours en retard, ou rétrospectivement, lorsqu'on s'en trouve affecté on ne sait pas comment. " (François Tosquelles dans Pratique de l'institutionnel et politique, op. cit., p. 95). 
57 Jacques Lacan, Le Séminaire de Jacques Lacan. Livre 3, Les Psychoses, 1955-1956; texte établi avec Jacques-Alain Miller, Paris, Seuil, 1981.

58 Dalí insistera toute sa vie sur le caractère objectif et cognitif du regard paranoïa-critique destiné à matérialiser les fantasmes et visions de l'artiste dans les formes des objets perçus dans la réalité. "Il s'agit, d'une part, d'un mode de connaissance irrationnelle du monde qui peut être adopté par tout regardeur et grâce auquel l'objet acquiert des significations inattendues"; et, d'autre part, d'un procédé de création qui consiste à retranscrire "fidèlement sur la toile tout ce qu'il voit au travers de son regard paranoïaque ", et qui "met en évidence, par un travail conscient et critique, le fantasme qui est latent dans les formes de l'objet ". C'est donc une forme de connaissance irrationnelle qui se base sur un délire d'interprétation du monde perçu, cette méthode impliquant, d'un côté, une disponibilité particulière de l'artiste au délire (donc à un état mental perturbé) et, de l'autre, une intervention critique qui donne au délire une forme systématique, minutieuse et rigoureuse. Voir: Astrid Ruffa, Salvator Dalí, Barcelona, Ediciones de la Central, Cuadernos-Arte, 2010, p. 30.

59 Sur la base de ce constat, on peut supposer que le modèle de psychose auquel se réfère Tosquelles (et tous les partisans de la psychiatrie institutionnelle) est indirectement de facture surréaliste, et plus spécialement d'obédience lacano-dalinienne, si I'on prend au sérieux l'hypothèse qui restitue à Dalí le rôle d'inspirateur auprès d'un jeune Lacan totalement séduit par le mouvement surréaliste. J'appuie mon hypothèse sur l'ouvrage de José Ferreira, DalíLacan. La rencontre. Ce que le psychanalyste doit au peintre, Paris, L'Harmattan, 2003.

60 Voir à ce sujet les thèses de Patrick Fuery, Madness and Cinema: Psychoanalysis, Spectatorship and Culture, Basingstoke/New York, Palgrave Macmillan, 2004.

61 Le film sera, en effet, montré à plusieurs reprises lors de projections privées avant d'être en exclusivité parisienne au cinéma La Pagode, permettant ainsi sa diffusion à une plus large audience. Succès critique et public, objet de controverses concernant l'éthique, tant des médecins que des réalisateurs du cinéma direct, il circulera également dans le milieu des festivals et des ciné-clubs (voir la fiche de programmation de la Fédération française des ciné-clubs), recevant le Prix du film
20/ La Fête prisonnière

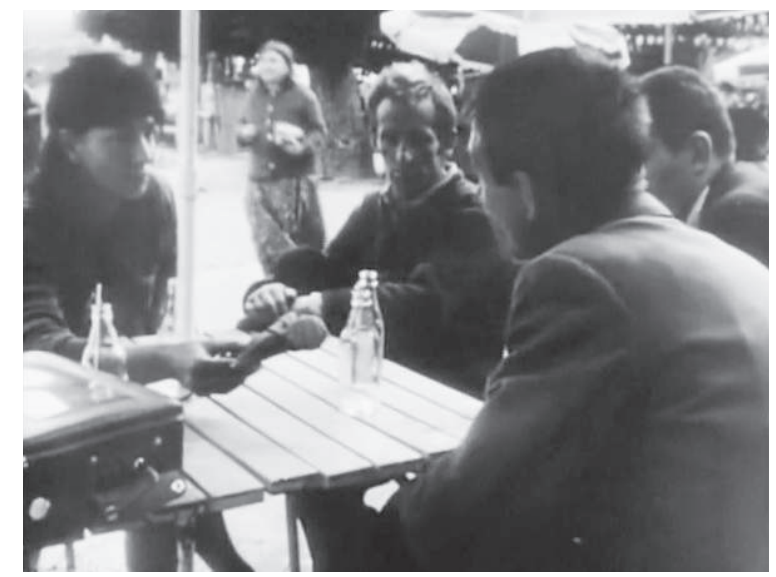

et à entendre, comme nul autre fou, un inconscient littéralement à ciel ouvert, selon l'analyse qu'en réalise Jacques Lacan dans son Séminaire (Livre 3, 1955-1956) 57. C'est autour de ce primat de la parole du fou que se rejoignent psychanalyse et surréalisme, et par voie de conséquence, le cinéma direct qui en "récolte les fruits» en quelque sorte. Prônant ensemble l'idée que le discours du psychotique a un sens, tout sujet parlant est invité à pénétrer dans son langage pour entendre ce qu'il a à nous dire sur l'inconscient. Or, ce point de jonction qui s'établit autour du sens du discours psychotique n'est pas pure coïncidence.

On l'ignore parfois, mais l'horizon intellectuel de Lacan a été grandement influencé par ses contacts avec le surréalisme et notamment par la méthode paranoïa-critique de Dalí qui consiste en une simulation de la pathologie psychotique permettant de connaître la réalité autrement et de faire partager cette connaissance à travers des formes objectives (fig. 21) 58. Les thèses lacaniennes sur la psychose sont donc marquées par cette source d'inspiration majeure, bien que dans sa thèse de doctorat, il peine à reconnaître cette dette intellectuelle 59. Ainsi, pour Lacan, le psychotique serait le fou le plus génial, le plus créatif, le plus inventif, mais aussi le plus "réaliste " puisqu'il mène le plus directement à la vérité de l'inconscient, à savoir au Réel de la psychose qui laisse littéralement l'inconscient à découvert. Si le film accueille ce langage de la folie sur le plan de son représenté, il le reçoit également au niveau d'un mode de fonctionnement qui travaille, comme la psychose, sur des absences de causalité, de logique et de signifiance immédiate, la pensée de type psychotique partageant avec le flux filmique une relative autonomie à l'égard de la réalité de l'expérience commune ${ }^{60}$. 
C’est probablement cette "vérité» de la psychose captée par l'équipe de Ruspoli - une vérité à la fois totalement déployée et insaisissable qui explique l'enthousiasme des psychiatres et psychanalystes vis-à-vis du film lors d'une projection privée le 13 juin 196261, comme le prouve cette observation du $\mathrm{D}^{\mathrm{r}}$ Henri Ey, l'un des psychiatres français les plus célèbres du XXe siècle:

"C’est l'image de mon expérience quotidienne... Ce film a une très grande valeur psychiatrique... Si j'avais la disposition de ce film dans mon service, je leur montrerai l'intérêt extraordinaire qui s'attache à la psychothérapie de cette masse si terrible qui garnit les hôpitaux à Bonneval comme à Saint-Alban. $\mathbf{6 2}$

Car contrairement à ce qu'avancent les historiens traditionnels du cinéma direct63, les professionnels, loin d'être déçus, ont déclaré la nécessité de faire circuler le film dans les milieux de la santé, sa dimension didactique et pédagogique faisant l'objet de tous les éloges. Une correspondance échangée cette année-là entre, d'une part, le société de production Argos et le $\mathrm{D}^{\mathrm{r}}$ Leroy (alors président de la Ligue d'Hygiène mentale) ${ }^{64}$, et, d'autre part, le $\mathrm{D}^{\mathrm{r}}$ Leroy et une représentante du Ministère de la Santé Publique, $\mathrm{M}^{\mathrm{lle}} \mathrm{M}$. R. Mamelet65, atteste en effet de l'intérêt porté par les médecins, psychanalystes et psychologues à ce film. La Société Argos Films souligne à cette occasion combien cette projection devant un parterre d'experts «nous a permis de constater sans

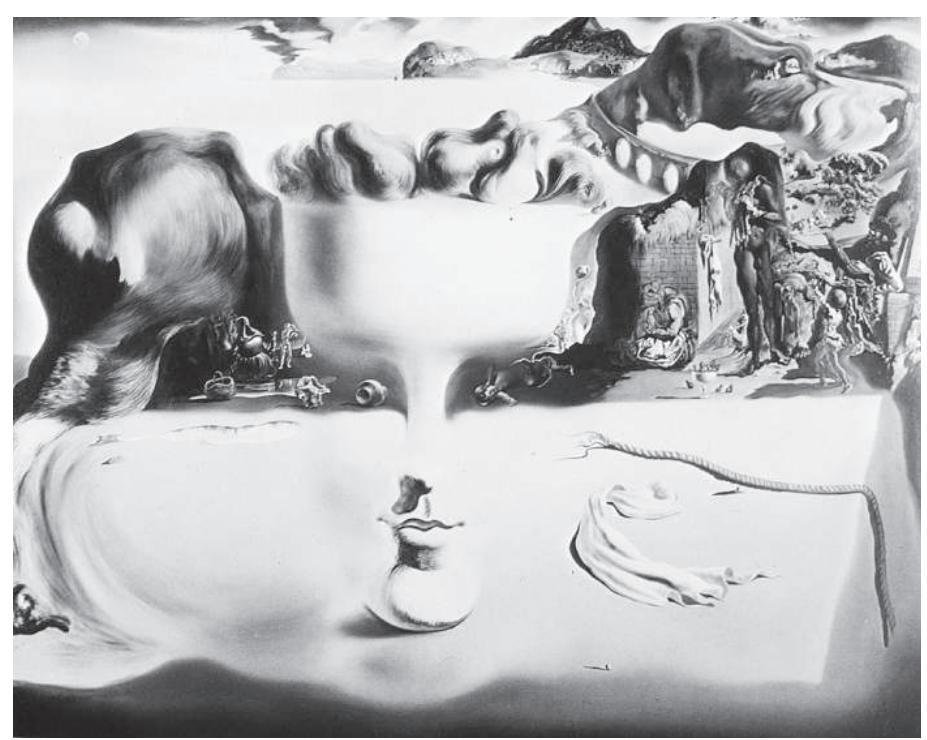

21/ Apparition d'un visage et d'un compotier sur une plage, Salvador Dalí, 1938 expérimental et d'avant-garde au Festival de Bergame 1962 et le prix de la qualité au Festival des Peuples 1962. Robert Benayoum dans France Observateur, notamment, soulève les aspects moraux du film: "Ici, la question peut se poser de savoir s'il est absolument irréprochable de fixer sur la pellicule les incohérences de certains esprits dérangés qui, un jour, reprendront leur place dans cette société faite, semble-t-il, de spectateurs et de voyeurs. [...] A travers les méandres babyloniens de l'asile, qu'une fête grinçante et gauche tente en vain de conjurer, les médecins sont peut-être les plus irrémédiablement enfermés dans leur rôle cumulatif d'enquêteur, de juge, de (parent) et de thérapeute." (20 septembre 1962).

62 Lettre du 14 juin 1962. Archives personnelles de Mario Ruspoli. Je remercie Séverine Graff pour avoir mis à ma disposition ces sources compilées dans le cadre de sa recherche personnelle autour du cinéma direct, ainsi que pour nos échanges à propos de Regard sur la folie.

63 Guy Marsolais estime que "les médecins de l'hôpital ont regretté que l'accent du film n'ait pas été mis sur l'aspect thérapeutique. Et ils ont raison." (Guy Marsolais, L'aventure du direct, op. cit., p. 236). Même son de cloche chez Gauthier, Pilard et Suchet, certainement induits en erreur par Marsolais: "Pour les professionnels (à qui il n'était pas forcément destiné), le film est un peu décevant, dans la mesure où il ne s'attarde pas sur les symptômes et les comportements." (Guy Gauthier, Philippe Pilard, Simone Suchet, Le Documentaire passe au direct, op. cit., p. 70). La réception critique contemporaine à la sortie du film prouve au contraire que les médecins s'y sont intéressés, comme le montre par exemple un entretien réalisé par Michel Vianey pour L'Express auprès d'un "jeune et brillant psychiatre" qui estime que "la valeur cinématographique de ce document est incontestable." (4 octobre 1962, p. 29).

64 Lettre du 14 juin 1962. Archives personnelles de Mario Ruspoli.

65 Lettre du 15 juin 1962 adressée par le $\mathrm{D}^{\mathrm{r}}$ Leroy à $\mathrm{M}^{\text {Ile }}$ Mamelet, Administrateur civil, chef du bureau de l'Hygiène sociale, à Paris. Archives personnelles de Mario Ruspoli. Le but de cette lettre consiste à faire acheter au Ministère de la Santé publique une copie du film, en s'appuyant sur l'argument de l'approbation des spécialistes suite à la projection du film. 
66 Lettre du 14 juin 1962, op. cit.

67 Lettre du $D^{r}$ Leroy datée du 15 juin 1962 et adressée au Ministère de la Santé Publique.

68 L'hôpital accueille en mai 1960, sous l'initiative de François Tosquelles, Hélène Chaigneau et Jean Oury, les premières journée du G.T.P.S.I (Groupe de Travail sur la Psychothérapie et la Sociothérapie Institutionnelles) qui rassembleront jusqu'en 1965, les acteurs principaux de ce mouvement. Ces journées semblent avoir laissé des traces indélébiles dans les mémoires et dans les cœurs, tant les débats furent passionnants et passionnés, le film de Ruspoli s'inscrivant dans ce climat intellectuel vif et intense. Voir Patrick Faugeras, L'ombre portée de François Tosquelles, op. cit., p. 70; Jean-Claude Polack, "Introduire la politique plus vivement", Chantier de mai 1968 à..., La Parole Errante à la Maison de l'Arbre, Montreuil (document pdf en ligne sur internet), p. 27.

69 Le cinéma croise la trajectoire de la psychiatrie institutionnelle, non seulement via le film de Ruspoli, mais également via: 1) Un projet de film sur le psychodrame, imaginé par Ruspoli d'entente avec le Dr Bour, médecin-chef de I'hôpital psychiatrique départemental du Pas-deCalais, prouvant ainsi la pérennité d'un intérêt réciproque entre les milieux du cinéma direct et celui de la psychiatrie (on trouve trace de ce projet dans les archives personnelles de Ruspoli, la correspondance afférente s'étalant du 5 août 1964 au 7 mai 1973). Le psychodrame propose une approche ludique des conflits psychiques que l'on met en scène dans le cadre de jeux de rôles supervisés par un thérapeute. Cette technique sera utilisée dans le cadre de la psychiatrie institutionnelle qui s'inspire notamment des travaux de Jacob Lévy Moreno. Voir: Anne Ancelin Schützenberger, Le psychodrame, Paris, Payot \& Rivages, 2003. 2) L'animation à Saint-Chély d'Apcher (près de SaintAlban) d'un ciné-club par François Tosquelles qui propose à un public affluent de discuter de sujets ayant trait à la psychopathologie. 3) La collaboration de Roger Gentis et de François Tosquelles au film réalisé par Ruspoli et son équipe en 1961, Les Inconnus de la terre, et dans lequel on voit les psychiatres prendre la place des interviewers/analystes. 4) Un court métrage écrit et dessiné par un groupe de malades et réalisé par René Laloux, psychiatre à la clinique de Cour Cheverny, avec la collaboration de Félix Guattari et Jean Oury: Les dents du singe (1960). Ce film est visible sur Dailymotion. équivoque l'adhésion, pleine, entière et unanime, des médecins au film de Mario Ruspoli (réalisé avec le concours du Docteur Tosquelles et de son équipe)»66. Il semblerait que cette unanimité des médecins trouve sa cause dans la manière dont Ruspoli a réussi à articuler, sans créer de disjonction, sa tendance à l'objectivité restituant une réalité sans l'enjoliver à une dimension didactique «involontaire» qui touche de très près des médecins sensibles à cet "effet secondaire» induit par l'image d'une vérité «nue», celle de la parole du fou.

C'est ce que peut laisser penser le commentaire du $\mathrm{D}^{\mathrm{r}}$ Leroy à propos des réactions de ses collègues face aux vertus pédagogiques du film:

«Le $\mathrm{D}^{\mathrm{r}}$ Henri Ey a donné la première impression générale en soulignant l'émotion qui se dégage de cette vision d'une psychiatrie à son état le plus brut, sans artifice de présentation ni souci de propagande. Il s'est en même temps demandé si la vérité, la brutalité presque des images, qui constituent à ses yeux toute la valeur du film, en feraient un très bon instrument de propagande pour le grand public. [...] $\mathrm{Au}$ cours de la discussion sur la valeur didactique du film, le $\mathrm{D}^{\mathrm{r}}$ Bernard a reconnu qu'il constituait <une mine d'observations d'une très grande valeur didactique qui pourrait faire prendre conscience à ses infirmiers et à ses élèves des problèmes qui se posent à eux >.»67

D’une manière générale, les médecins et psychothérapeutes ayant vu le film attirent l'attention sur l'appréhension sincère et prévenante d'une réalité qui mérite d'être montrée tant aux spécialistes de la branche qu'aux profanes qui méconnaissent la maladie mentale et les lieux où elle se soigne. Le didactisme du Regard sur la folie, cependant, ne relève ni d'une clarification édifiante ni d'une authentification obscène, mais bien d'une sorte de cohésion et de solidarité toujours respectueuses entre une pratique filmique et son objet d'étude. Les entretiens donnés par ceux et celles qui ont œuvré dans le champ de la psychiatrie institutionnelle, au moment du tournage et bien des années après celui-ci, témoignent de l'importance qu'a eu ce film dans leur formation intellectuelle, non seulement parce qu'il a été réalisé à un moment particulièrement intense de l'histoire du mouvement ${ }^{68}$, mais certainement aussi en raison de ses corrélations formelles et éthiques avec celui-ci. Remettant en question les pouvoirs, les hiérarchies et les règles, à contrecourant des pratiques dominantes, le cinéma direct et la psychiatrie institutionnelle se rejoignent, grâce à ce film, sur un terrain permettant d'appliquer des compétences complémentaires centrées sur le regard et l'écoute de la folie69. 